\title{
To Ba or not to Ba: Enrichment in s-process elements in binary systems with WD companions of various masses
}

\author{
T. Merle ${ }^{1}$, A. Jorissen ${ }^{1}$, S. Van Eck ${ }^{1}$, T. Masseron ${ }^{1,2}$, and H. Van Winckel ${ }^{3}$ \\ 1 Institut d'Astronomie et d'Astrophysique, Université Libre de Bruxelles, CP 226, Boulevard du Triomphe, 1050 Brussels, Belgium \\ e-mail: tmerle@ulb.ac.be \\ 2 Institute of Astronomy, University of Cambridge, Madingley Road, Cambridge CB3 OHA, UK \\ ${ }^{3}$ Instituut voor Sterrenkunde, Katholieke Universiteit Leuven, Celestijnenlaan 200D, 3200 Heverlee, Belgium
}

Received 12 July 2015 / Accepted 16 October 2015

\begin{abstract}
Context. The enrichment in s-process elements of barium stars is known to be due to pollution by mass transfer from a companion formerly on the thermally pulsing asymptotic giant branch (AGB), now a carbon-oxygen white-dwarf (WD).

Aims. We are investigating the relationship between the $s$-process enrichment in the barium star and the mass of its WD companion. It is expected that helium WDs, which have masses lower than about $0.5 M_{\odot}$ and never reached the AGB phase, should not pollute their giant companion with $s$-process elements. Therefore the companion should never turn into a barium star.

Methods. Spectra with a resolution of $R \sim 86000$ were obtained with the HERMES spectrograph on the $1.2 \mathrm{~m}$ Mercator telescope for a sample of 11 binary systems involving WD companions of various masses. We used standard 1D local thermodynamical equilibrium MARCS model atmospheres coupled with the Turbospectrum radiative-transfer code that is embedded in the BACCHUS pipeline to derive the atmospheric parameters through equivalent widths of Fe I and Fe II lines. Least-squares minimization between the observed and synthetic line shape was used to derive the detailed chemical abundances of CNO and s-process elements.

Results. The abundances of $s$-process elements for the entire sample of 11 binary stars were derived homogeneously. The sample encompasses all levels of overabundances: from solar $[s / \mathrm{Fe}]=0$ to $1.5 \mathrm{dex}$ in the two binary systems with S-star primaries (for which dedicated MARCS model atmospheres were used). The primary components of binary systems with a WD more massive than $0.5 M_{\odot}$ are enriched in $s$-process elements. We also found a trend of increasing $[s / \mathrm{Fe}]$ with $[\mathrm{C} / \mathrm{Fe}]$ or $[(\mathrm{C}+\mathrm{N}) / \mathrm{Fe}]$.

Conclusions. Our results confirm the expectation that binary systems with WD companions less massive than $0.5 M_{\odot}$ do not host barium stars.
\end{abstract}

Key words. stars: abundances - white dwarfs - stars: late-type - binaries: spectroscopic

\section{Introduction}

The puzzle of the barium stars, a class of $\mathrm{K}$ giants with overabundances of heavy elements produced by the $s$-process of nucleosynthesis (Bidelman \& Keenan 1951), has been solved by McClure et al. (1980), who unravelled the binary nature of these stars, and made it clear that the companion is responsible for the heavy-element synthesis. This solution of course requires the companion to be a carbon-oxygen white dwarf (WD), which synthesized the heavy elements while on the thermally pulsing asymptotic giant branch (TP-AGB). An indirect argument supporting this hypothesis has been provided by McClure $\&$ Woodsworth (1990) and Jorissen et al. (1998) in the form of the mass-function distribution, which is consistent with the companion masses peaking at $0.6 M_{\odot}$, as expected for carbon-oxygen WDs. It is only quite recently that a clear direct argument was presented by Gray et al. (2011) in the form of UV excess flux from these systems detected by GALEX that is attributable to a warm WD. Earlier studies by Schindler et al. (1982), Dominy \& Lambert (1983), Böhm-Vitense et al. (1984, 2000), Jorissen et al. (1996), and Frankowski \& Jorissen (2006) found similar evidence, although somewhat less convincingly or for a smaller set.

With the advent of sensitive far-UV and X-ray satellites such as EUVE, XMM-Newton, and ROSAT, many warm WDs were discovered from their X-ray or UV flux (see Bilíková et al. 2010, and references therein). Some of them are members of binary systems involving a late-type primary star (e.g. Landsman et al. 1993; Vennes et al. 1995, 1997, 1998; Jeffries \& Smalley 1996; Jorissen et al. 1996; Christian et al. 1996; Burleigh et al. 1997, 1998; Hoard et al. 2007; Bilíková et al. 2010), with the latter thus being barium-star candidates. Of course, for this to occur, the progenitor of the current WD had to be a TP-AGB star with an atmosphere enriched in $s$-process elements. This in turn requires that the $s$-process nucleosynthesis operated in the $\mathrm{H}$ - and He-burning intershell region and that the $s$-process material was dredged up to the surface. The currently available sample of WDs in binary systems offers an interesting way to test the above paradigm, especially when the mass of the WD is known. By checking whether or not the current companion of the WD is enriched in $s$-process elements, we may trace the operation of $s$ process and dredge-up in the AGB progenitor of the current WD. Since its mass is equivalent to the AGB core mass at the end of its evolution, we may thus set constraints on models of AGB evolution by investigating whether there is a threshold on the WD mass for its companion to become a barium star. It is expected for instance that He WDs, which are less massive than CO WDs, had progenitors that did not ascend the AGB. Their companions should thus never be barium stars. This was recently proven to be true for the IP Eri system, whose K primary star, companion of a He WD, is not a barium star (Merle et al. 2014). The present paper addresses this question on a larger sample of binaries involving WDs covering a broad range of masses. 
Table 1. Sample of binary systems with a WD companion and known orbital elements.

\begin{tabular}{|c|c|c|c|c|c|c|}
\hline Names & Spectral type & $M_{\mathrm{WD}}\left(M_{\odot}\right)$ & $P(\mathrm{~d})$ & $e$ & $f\left(M_{1}, M_{2}\right)\left(M_{\odot}\right)$ & Ref. \\
\hline $\begin{array}{l}\text { EUVE J0459-102 } \\
\text { HR } 1608 \text { (63 Eri) }\end{array}$ & G4V + He WD & 0.3 & $903 \pm 5$ & $0.30 \pm 0.06$ & $0.009 \pm 0.003$ & 2,3 \\
\hline HD 185510 (V1379 Aql) & $\mathrm{K} 0 \mathrm{III}+\mathrm{sdB}$ & $0.30-0.36$ & $20.66187 \pm 0.00058$ & $0.094 \pm 0.011$ & $0.0042 \pm 0.0002$ & 1 \\
\hline $\begin{array}{l}\text { EUVE J0254-053 } \\
\text { HD } 18131 \text { (IP Eri) }\end{array}$ & K0 IV + He WD & 0.4 & $1071.0 \pm 1.8$ & $0.25 \pm 0.01$ & $0.0036 \pm 0.0001$ & 4,5 \\
\hline $\begin{array}{l}\text { EUVE J0515+326 } \\
\text { HD 33959C (14 Aur C) }\end{array}$ & F3V & $0.53-0.69$ & $2.99322 \pm 0.00005$ & 0 & $0.0079 \pm 0.0002$ & 3 \\
\hline $\begin{array}{l}\text { EUVE J1732+74.2 } \\
\text { HD } 160538 \text { (DR Dra) }\end{array}$ & K0III & 0.55 & $903.8 \pm 0.4$ & $0.07 \pm 0.03$ & $0.0035 \pm 0.0003$ & 1,17 \\
\hline HD 35155 (V1261 Ori) & $\mathrm{S} 4,1$ & $0.55 \pm 0.05$ & $640.7 \pm 2.7$ & $0.07 \pm 0.03$ & $0.032 \pm 0.003$ & 6,7 \\
\hline $\begin{array}{l}\text { 1RXS J151824.6+203423 } \\
\text { HR } 5692\end{array}$ & G8III Ba0.3 & $0.59-0.79$ & $506.45 \pm 0.18$ & $0.3353 \pm 0.0056$ & $0.0112 \pm 0.0003$ & 6,8 \\
\hline HD 121447 (IT Vir) & K7III Ba SC2 & $0.5-0.7$ & $185.66 \pm 0.07$ & 0.0 & $0.025 \pm 0.001$ & 16 \\
\hline HD 202109 ( $\zeta$ Cyg) & G8III Ba0.6 & $0.7-1.1$ & $6489 \pm 31$ & $0.22 \pm 0.03$ & $0.023 \pm 0.003$ & $9,10,14$ \\
\hline $\begin{array}{l}\text { RX J2307.1+2528 } \\
\text { HR } 8796 \text { (56 Peg) }\end{array}$ & K0Iab Ba & $0.75-1.15$ & $111.14 \pm 0.01$ & 0 & $(3.7 \pm 0.3) 10^{-5}$ & $11,12,13$ \\
\hline $\begin{array}{l}\zeta \text { Cap } \\
\text { HD } 204075\end{array}$ & G4Ib Ba & 1 & $2378 \pm 55$ & $0.28 \pm 0.07$ & $0.004 \pm 0.001$ & 14,15 \\
\hline
\end{tabular}

Notes. $P$ and $e$ are the orbital period and eccentricity and $f\left(M_{1}, M_{2}\right)$ is the mass function, ordered by increasing WD mass.

References. (1) Fekel et al. (1993); (2) Landsman et al. (1993); (3) Vennes et al. (1998) (4) Burleigh et al. (1997); (5) Merle et al. (2014); (6) Jorissen et al. (1996); (7) Jorissen et al. (1992); (8) Stefanik et al. (2011); (9) Dominy \& Lambert (1983); (10) Griffin \& Keenan (1992); (11) Schindler et al. (1982); (12) Griffin (2006); (13) Frankowski \& Jorissen (2006); (14) Böhm-Vitense (1980); (15) Jorissen et al. (1998); (16) Jorissen et al. (1995); (17) Fekel \& Simon (1985).

The paper is organized as follows. Section 2 describes the stellar sample and Sect. 3 the observations. Section 4 presents the method used for deriving the atmospheric parameters, and Sect. 5 the resulting abundances, separately for $\mathrm{CNO}$ and $s$-process elements. Sections 6 and 7 contain the discussion of these results and our conclusions.

\section{Sample}

The binary systems we selected have WD components orbiting a cool star (with $T_{\text {eff }}<7000 \mathrm{~K}$ ). The main selection criterion was the availability of the WD mass, which ranges from 0.3 to $1.0 M_{\odot}$. Our sample is listed in Table 1 . The sample also covers a broad range of orbital periods and eccentricities. The WD masses are taken from the references listed in Table 1.

Here follow remarks on some targets of our sample.

IP Eri (HD 18131) consists of a K0 IV star and a He WD companion. Its evolutionary history has been studied by Siess et al. (2014), and an abundance study was performed by Merle et al. (2014).

14 Aur C (HD 33959) is member of a quadruple system whose component $\mathrm{A}$ is a $\delta$ Scuti star. 14 Aur $\mathrm{C}$ is located $14.6 \operatorname{arcsec}$ from A, at a distance of $104 \pm 32 \mathrm{pc}$ from the Sun (van Leeuwen 2007). Spectral types F2V+DA (Vennes et al. 1998) or F5V (Gray et al. 2003) have been proposed. It appears to be a fast rotator. We derived for this $\operatorname{star} v \sin i=16 \mathrm{~km} \mathrm{~s}^{-1}$. No atmospheric parameters are available yet in the literature for that star. The orbital period of only $3 \mathrm{~d}$ makes the dwarf star
14 Aur $\mathrm{C}$ an outlier in the period distribution of giant barium stars, but not in that of dwarf carbon stars (if 14 Aur C is one such), where at least one star with a similar period is known (HE 0024-2523 has a period of $3.4 \mathrm{~d}$; Lucatello et al. 2003).

V1261 Ori (HD 35155) is a symbiotic S star (Ake et al. 1991; Jorissen et al. 1996).

IT Vir (HD 121447) is a border case between barium and S stars, since it has been classified as SC2 (Ake 1979), K4Ba (Abia \& Wallerstein 1998), K7Ba, and S0.

$\zeta$ Cap (HD 204075) is a prototype barium star.

DR Dra (HD 160538) and V1379 Aql (HD 185510) have strong emission cores in the $\mathrm{Ca}$ II $\mathrm{H}$ and $\mathrm{K}$ lines (Fig. 1), indicative of chromospheric activity related to rapid rotation, as confirmed by our analysis $\left(v \sin i=8.5 \mathrm{~km} \mathrm{~s}^{-1}\right.$ and $18 \mathrm{~km} \mathrm{~s}^{-1}$, respectively; see Fig. 3) in agreement with the results of Fekel et al. (1993). Emission is also visible in the IR Ca II triplet.

\section{Observations}

High-resolution spectra $(R \sim 86000)$ were obtained using the HERMES spectrograph (Raskin et al. 2011) operating at the $1.2 \mathrm{~m}$ telescope Mercator at La Palma (Canary Islands, Spain). The HERMES spectrograph is a fiber-fed echelle spectrograph that provides a full spectral coverage from 377 to $900 \mathrm{~nm}$. All the spectra were reduced using the HERMES pipeline software and have a typical signal-to-noise ratio of about 200. The observations were carried out between June 2009 and September 2014. 


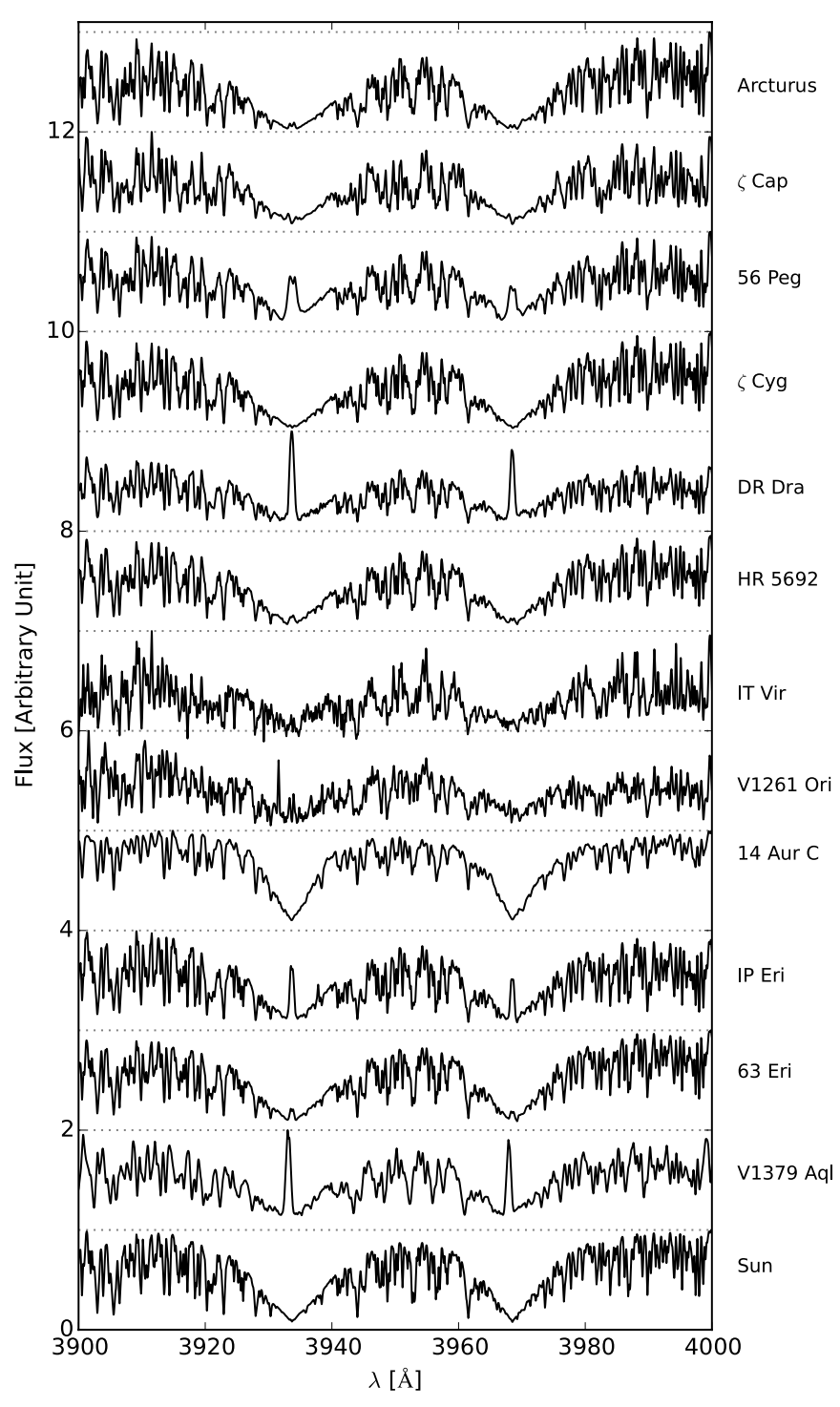

Fig. 1. $\mathrm{Ca} I \mathrm{II} \mathrm{H}$ and $\mathrm{K}$ lines for the sample stars. For comparison, the spectra of the Sun and Arcturus are plotted at the bottom and top of the figure, respectively. Evidence of activity is present in 63 Eri, IP Eri, V1379 Aql, DR Dra, and 56 Peg.

\section{Atmospheric parameters}

First guesses for the atmospheric parameters were determined from previous works (as listed in Sect. 2), from photometry or spectral classification. We assumed the metallicity to be solar to start with [the solar abundance set from Grevesse et al. (2007) is used throughout this paper, in particular with $\left.A_{\odot}(\mathrm{Fe})=7.45^{1}\right]$.

The atmospheric parameters were then determined iteratively using the pipeline BACCHUS developed by one of the authors (TMa; see also Jofre et al. 2014). This pipeline is based on the 1D local thermodynamical equilibrium (LTE) spectrumsynthesis code Turbospectrum (Alvarez \& Plez 1998; Plez 2012) and the method for deriving the atmospheric parameters is based on the fit of the equivalent widths of Fe I and Fe II lines. The code automatically derives the effective temperature $T_{\text {eff }}$, the logarithmic surface gravity $\log g$, the global metallicity $[\mathrm{Fe} / \mathrm{H}]$, and the microturbulent velocity $\xi$ by removing any trend in the

\footnotetext{
1 Defined as $A(\mathrm{Fe})=\log [n(\mathrm{Fe}) / n(\mathrm{H})]+12$, where $n(\mathrm{Fe})$ and $n(\mathrm{H})$ are the number densities of $\mathrm{Fe}$ and $\mathrm{H}$.
}

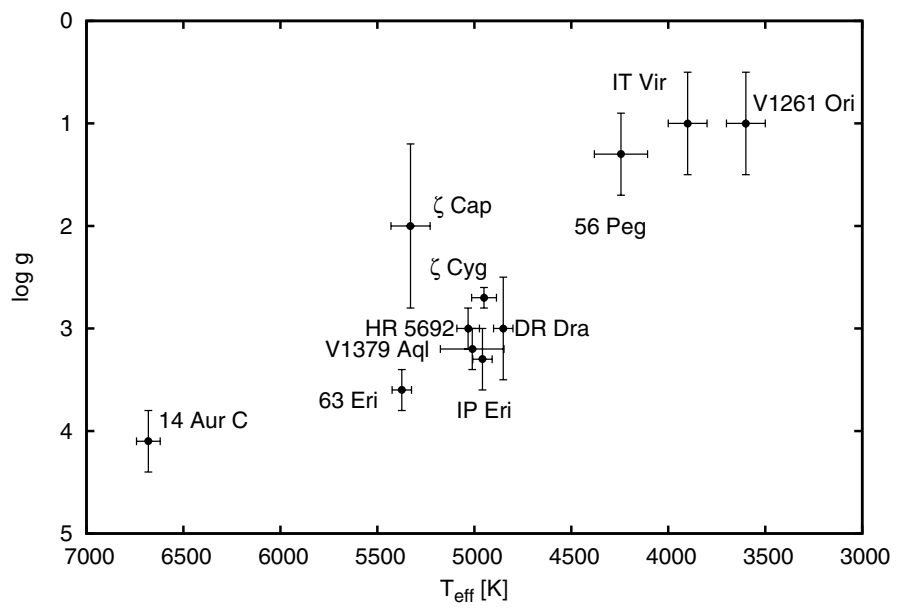

Fig. 2. Surface gravity vs. effective temperature for the sample stars.

$[\mathrm{Fe} / \mathrm{H}]$ vs. $\chi$ and $[\mathrm{Fe} / \mathrm{H}]$ vs. $W / \lambda$ relations (where $\chi$ is the energy of the lower level of a line, $W$ the measured equivalent width, and $\lambda$ the wavelength of the considered line), and by forcing lines of Fe I and Fe II to yield the same abundance. We used MARCS model atmospheres (Gustafsson et al. 2008) and a line list constructed from the VALD3 database (Kupka et al. 2011), complemented for Fe and V lines by data from Den Hartog et al. (2014b,a) and Ruffoni et al. (2014).

The synthetic spectra were convolved with a Gaussian function with a $\sigma$ varying from 6 to $12 \mathrm{~km} \mathrm{~s}^{-1}$ to match the stellar macroturbulence and/or HERMES resolution. Only reduced equivalent widths $(W / \lambda)$ of Fe I and Fe II lines lower than 0.025 are retained in the analysis. For the fast rotators 14 Aur C $\left(V \sin i=16 \mathrm{~km} \mathrm{~s}^{-1}\right)$ and $\mathrm{V} 1379 \operatorname{Aql}\left(V \sin i=18 \mathrm{~km} \mathrm{~s}^{-1}\right)$ we convolved the synthetic spectra with a rotational profile.

Merle et al. (2014) performed an abundance analysis of IP Eri and obtained the following atmospheric parameters: $T_{\text {eff }}=$ $4960 \pm 100 \mathrm{~K}, \log g=3.3 \pm 0.3$, and $[\mathrm{Fe} / \mathrm{H}]=+0.09 \pm 0.08$, which are used here as well.

The atmospheric parameters of the prototypical Ba star $\zeta$ Cap $\left(T_{\text {eff }}=5397 \pm 82 \mathrm{~K}, \log g=1.48 \pm 0.16\right.$, and $[\mathrm{Fe} / \mathrm{H}]=$ $-0.14 \pm 0.10)$ were derived by Prugniel et al. (2011). They were rederived in the present study, and the two parameter sets are similar to each other.

For the two S stars (IT Vir and V1261 Ori) the atmospheric parameters cannot be derived with BACCHUS because strong molecular lines blend many Fe lines and prevent using their equivalent widths. Instead, for V1261 Ori, we adopted the parameters derived by Neyskens et al. (2015), who took the specific chemical composition of $\mathrm{S}$ stars and its feedback on the model-atmosphere structure into account. A new grid of MARCS model atmospheres dedicated to S stars (Van Eck et al. 2011) has been used for that purpose. Neyskens et al. (2015) obtained $T_{\text {eff }}=3600 \mathrm{~K}, \log g=1.0,[\mathrm{Fe} / \mathrm{H}]=-0.5, \mathrm{C} / \mathrm{O}=0.5$, and $[s / \mathrm{Fe}]=+1.0$. These parameters are adopted here.

For IT Vir, a border case between Ba and S stars, Abia \& Wallerstein (1998) estimated the atmospheric parameters to be $T_{\text {eff }}=4000 \mathrm{~K}, \log g=1.0$, and $[\mathrm{Fe} / \mathrm{H}]=-1.0$ with $\xi=2 \mathrm{~km} \mathrm{~s}^{-1}$ and $\mathrm{C} / \mathrm{O}=0.95$, while Smith (1984) had previously found $T_{\text {eff }}=4200 \mathrm{~K}, \log g=0.8,[\mathrm{Fe} / \mathrm{H}]=0.05$, and $\mathrm{C} / \mathrm{O}=0.79$. The $V-K$ index $(V-K=3.65)$ combined with the calibration of Bessell et al. (1998) yields $T_{\text {eff }}=3900 \mathrm{~K}$. We adopted this value for the temperature combined with the gravity reported by Abia \& Wallerstein (1998). 
Table 2. Spectroscopic stellar parameters derived with BACCHUS.

\begin{tabular}{|c|c|c|c|c|c|}
\hline Star name & HD & $T_{\mathrm{eff}}(\mathrm{K})$ & $\log g$ & {$[\mathrm{Fe} / \mathrm{H}]$} & $\xi\left(\mathrm{km} \mathrm{s}^{-1}\right)$ \\
\hline V1261 Ori ${ }^{a}$ & 35155 & $3600 \pm 100$ & $1.0 \pm 0.5$ & $-0.5 \pm 0.5$ & $2.0 \pm 0.5$ \\
\hline IT $\operatorname{Vir}^{a}$ & 121447 & $3900 \pm 100$ & $1.0 \pm 0.5$ & $-0.5 \pm 0.5$ & $2^{b}$ \\
\hline 56 Peg & 218356 & $4244 \pm 137$ & $1.3 \pm 0.4$ & $-0.45 \pm 0.12$ & $1.55 \pm 0.06$ \\
\hline DR Dra & 160538 & $4851 \pm 50$ & $3.0 \pm 0.5$ & $-0.14 \pm 0.10$ & $1.76 \pm 0.05$ \\
\hline$\zeta$ Cyg & 202109 & $4950 \pm 64$ & $2.7 \pm 0.1$ & $-0.03 \pm 0.08$ & $1.43 \pm 0.03$ \\
\hline IP Eri & 18131 & $4958 \pm 50$ & $3.3 \pm 0.3$ & $0.09 \pm 0.08$ & $1.45 \pm 0.04$ \\
\hline V1379 Aql & 185510 & $5011 \pm 164$ & $3.2 \pm 0.2$ & $-0.23 \pm 0.12$ & $1.68 \pm 0.17$ \\
\hline HR 5692 & 136138 & $5032 \pm 58$ & $3.0 \pm 0.2$ & $-0.08 \pm 0.09$ & $1.35 \pm 0.04$ \\
\hline$\zeta$ Cap & 204075 & $5329 \pm 101$ & $2.0 \pm 0.8$ & $-0.08 \pm 0.13$ & $2.26 \pm 0.05$ \\
\hline 63 Eri & 32008 & $5374 \pm 50$ & $3.6 \pm 0.2$ & $-0.18 \pm 0.08$ & $1.03 \pm 0.04$ \\
\hline 14 Aur C & $33959 \mathrm{C}$ & $6681 \pm 61$ & $4.1 \pm 0.3$ & $-0.11 \pm 0.08$ & $1.75 \pm 0.09$ \\
\hline
\end{tabular}

Notes. Stars are ordered according to increasing $T_{\text {eff }}{ }^{(a)}$ Atmospheric parameters not determined with BACCHUS. ${ }^{(b)}$ No uncertainty given by Abia \& Wallerstein (1998) from whom this value has been adopted.

Table 3. C, N, and $\mathrm{O}$ abundances.

\begin{tabular}{|c|c|c|c|c|c|}
\hline Star & {$[\mathrm{Fe} / \mathrm{H}]$} & {$[\mathrm{C} / \mathrm{Fe}]$} & {$[\mathrm{N} / \mathrm{Fe}]$} & {$[\mathrm{O} / \mathrm{Fe}]$} & $\mathrm{C} / \mathrm{O}$ \\
\hline V1261 Ori ${ }^{a}$ & $-0.66 \pm 0.09$ & $0.39 \pm 0.14^{1}$ & $0.73 \pm 0.11^{2}$ & $0.54 \pm 0.08^{3}$ & 0.38 \\
\hline \multirow[t]{2}{*}{$\mathrm{IT}_{\mathrm{Vir}^{b}}$} & $-0.45 \pm 0.25$ & 0.55 & $1.39 \pm 0.30(50)$ & 0.40 & 0.75 \\
\hline & & 0.62 & $1.11 \pm 0.20(94)$ & 0.60 & 0.90 \\
\hline $56 \mathrm{Peg}$ & $-0.45 \pm 0.12$ & $0.01 \pm 0.23(13)$ & $-0.17 \pm 0.07(82)$ & $0.12 \pm \quad-\quad(1)$ & 0.63 \\
\hline DR Dra & $-0.14 \pm 0.10$ & $-0.23 \pm 0.07$ & $-0.21 \pm 0.09(82)$ & $0.15 \pm \quad-\quad(1)$ & 0.39 \\
\hline$\zeta$ Cyg & $-0.03 \pm 0.08$ & $-0.22 \pm 0.13$ & $0.11 \pm 0.05$ & $0.16 \pm 0.06$ & 0.44 \\
\hline IP Eri & $0.09 \pm 0.08$ & $-0.14 \pm 0.16(18)$ & $-0.23 \pm 0.06(96)$ & $0.14 \pm \quad-\quad(1)$ & 0.54 \\
\hline V1379 Aql & $-0.23 \pm 0.12$ & $-0.24 \pm 0.13$ & $0.21 \pm 0.05(29)$ & - & - \\
\hline HR 5692 & $-0.08 \pm 0.09$ & $-0.24 \pm 0.10$ & $-0.16 \pm 0.08(90)$ & $0.20 \pm \quad-\quad(1)$ & 0.43 \\
\hline$\zeta$ Сар & $-0.08 \pm 0.13$ & $0.11 \pm 0.05(16)$ & $0.38 \pm 0.07(85)$ & $0.12 \pm 0.08$ & 0.79 \\
\hline 63 Eri & $-0.18 \pm 0.08$ & $-0.12 \pm 0.18$ & $0.25 \pm 0.05(50)$ & $0.31 \pm 0.04(6)$ & 0.20 \\
\hline 14 Aur C & $-0.11 \pm 0.08$ & $0.22 \pm 0.03$ & $0.25 \pm 0.13$ & $0.16 \pm \quad-\quad(1)$ & 0.72 \\
\hline
\end{tabular}

Notes. Only the statistical uncertainties are given here. The number of lines used are given in parentheses. ${ }^{(a)}$ For this star, we used the Fe abundance from Kovacs (1983) scaled to the solar value of Holweger (1979). CNO abundances are taken from Smith \& Lambert (1990) and are relative to $\alpha$ Tau. ${ }^{(b)}$ Two equally likely abundance sets are listed for IT Vir (see text). ${ }^{(1)}$ From CO lines at 1.6 and $2.2 \mu \mathrm{m}$; ${ }^{(2)}$ from CN lines at $2.2 \mu \mathrm{m}$; (3) from $\mathrm{OH}$ lines at 1.6 and $2.2 \mu \mathrm{m}$.

The resulting atmospheric parameters are shown in Table 2, sorted by increasing effective temperatures (as in all the following tables). The uncertainties on the atmospheric parameters provided by the BACCHUS pipeline are those resulting from the propagation of the line-to-line $\mathrm{Fe}$-abundance scatter on the trends used to derive the atmospheric parameters, as explained above. Uncertainties on $T_{\text {eff }}$ lower than $50 \mathrm{~K}$ were arbitrarily set to a minimum value of $50 \mathrm{~K}$.

\section{Abundances}

We have chosen to perform the abundance analysis using spectrum synthesis. The detailed abundance analysis was performed using the BACCHUS pipeline abundance module. Only the least blended lines were retained for the analysis. This selection was performed over the whole HERMES wavelength range (i.e. [370-890] nm). The atomic line list (Table A.1) includes the isotopic shifts for Ba II (with an update for isotopes 130 and 132) and the hyperfine structure for La II from Masseron (2006). The $\mathrm{CH}$ molecular line list was taken from Masseron et al. (2014) and CN from Sneden et al. (2014). The references for the other molecular line lists ( $\mathrm{TiO}, \mathrm{SiO}, \mathrm{VO}, \mathrm{C}_{2}, \mathrm{NH}, \mathrm{OH}, \mathrm{MgH}, \mathrm{SiH}$, $\mathrm{CaH}$, and $\mathrm{FeH}$ ) can be found in Gustafsson et al. (2008). Line fitting was based on a least-squares minimization method, and all lines were visually inspected to check for possible problems (caused by e.g. line blends or cosmic hits). Results are presented in Table 3 for $\mathrm{CNO}$ abundances and in Tables 4 and 5 for $\mathrm{Sr}$, Y, $\mathrm{Zr}, \mathrm{Ba}, \mathrm{La}$, and $\mathrm{Ce}$. The uncertainties given in Tables 3 and 4 correspond to the standard deviation of the line-to-line dispersion, and in Table 5 we list the total uncertainties (including the effect of the uncertainties from the model parameters).

\section{1. $C, N, O$ abundances}

The $\mathrm{C}, \mathrm{N}$, and $\mathrm{O}$ abundances were derived in the following sequence: first $\mathrm{O}$, then $\mathrm{C}$, and finally $\mathrm{N}$. To derive the oxygen abundance, we mainly used the [O I] 630.030 and $636.378 \mathrm{~nm}$ lines since the OI triplet resonance line at $777 \mathrm{~nm}$ is strongly affected by NLTE effects [see Asplund (2005) for a detailed discussion]. The O I triplet line yields an LTE abundance larger by about $\sim 0.2-0.4$ dex than the abundance derived from the forbidden lines. This is not the case for the star 63 Eri, however, since for that star the triplet and the strong doublet at 844.636 and $844.676 \mathrm{~nm}$ give consistent abundances within 0.04 dex, even though the [OI] $630.030 \mathrm{~nm}$ is blended with a telluric line in its blue wing (which has therefore not been considered in the fit procedure). This star has one of the highest gravities, leading to a decrease of the non-LTE effects on the $777 \mathrm{~nm}$ triplet. For 14 Aur C, the two forbidden lines are lost in the noise, so that we instead used the highly excited very weak line at $615.68 \mathrm{~nm}$.

Although it is particularly difficult to find unblended atomic lines of CI, we found five useful weak lines (as listed in 
T. Merle et al.: To Ba or not to Ba?

Table 4. Absolute $s$-process element abundances $A(\mathrm{X})$ (defined as $A(\mathrm{X})=\log [n(\mathrm{X}) / n(\mathrm{H})]+12$, where $n(\mathrm{X})$ and $n(\mathrm{H})$ are the number densities of $\mathrm{X}$ and $\mathrm{H})$.

\begin{tabular}{|c|c|c|c|c|c|c|}
\hline Star & $A(\mathrm{Sr})$ & $A(\mathrm{Y})$ & $A(\mathrm{Zr})$ & $A(\mathrm{Ba})$ & $A(\mathrm{La})$ & $A(\mathrm{Ce})$ \\
\hline V1261 Ori & - & $2.66 \pm 0.27$ & $3.48 \pm 0.17$ & $3.02 \pm 0.32$ & $1.91 \pm 0.06$ & $2.33 \pm 0.07$ \\
\hline IT Vir & $3.44 \pm-$ & $2.70 \pm 0.12$ & $3.99 \pm 0.26$ & $4.05 \pm 0.18$ & $2.42 \pm 0.23$ & $2.52 \pm 0.20$ \\
\hline 56 Peg & $3.05 \pm 0.06(2)$ & $2.22 \pm 0.22$ & $2.44 \pm 0.07$ & $3.18 \pm 0.18$ & $1.12 \pm 0.09$ & $1.41 \pm 0.09$ \\
\hline DR Dra & $3.04 \pm 0.05(2)$ & $2.13 \pm 0.22$ & $2.77 \pm 0.06$ & $2.46 \pm 0.08$ & $1.17 \pm 0.10$ & $1.56 \pm 0.07$ \\
\hline$\zeta$ Cyg & $3.33 \pm \quad-\quad(1)$ & $2.61 \pm 0.16$ & $2.96 \pm 0.20$ & $3.16 \pm 0.25$ & $1.52 \pm 0.08$ & $1.87 \pm 0.12$ \\
\hline IP Eri & $3.14 \pm-$ & $2.06 \pm 0.12$ & $2.86 \pm 0.20$ & $2.53 \pm 0.09$ & $1.25 \pm 0.17$ & $1.60 \pm 0.09$ \\
\hline V1379 Aql & $2.89 \pm-$ & $2.23 \pm 0.04$ & $2.59 \pm 0.02$ & $2.29 \pm 0.07$ & $1.11 \pm 0.05$ & $1.52 \pm 0.16$ \\
\hline HR5692 & $3.16 \pm-$ & $2.63 \pm 0.20$ & $3.14 \pm 0.19$ & $2.90 \pm 0.07$ & $1.49 \pm 0.10$ & $1.77 \pm 0.13$ \\
\hline$\zeta$ Cap & $3.86 \pm 0.06(2)$ & $3.50 \pm 0.21$ & $3.87 \pm 0.28$ & $4.06 \pm 0.13$ & $2.09 \pm 0.10(11)$ & $2.47 \pm 0.15(10)$ \\
\hline 63 Eri & $2.84 \pm 0.01(2)$ & $2.06 \pm 0.13(10)$ & $2.48 \pm 0.17$ & $2.64 \pm 0.22$ & $1.15 \pm 0.06(10)$ & $1.55 \pm 0.06$ \\
\hline 14 Aur C & $3.04 \pm 0.05$ & $2.03 \pm 0.12$ & $2.64 \pm 0.07$ & $2.60 \pm 0.16$ & $1.22 \pm 0.11$ & $1.50 \pm 0.20$ \\
\hline $\operatorname{Sun}^{a}$ & $2.92 \pm 0.05$ & $2.21 \pm 0.02$ & $2.58 \pm 0.02$ & $2.17 \pm 0.07$ & $1.13 \pm 0.05$ & $1.70 \pm 0.10$ \\
\hline
\end{tabular}

Notes. Only the statistical uncertainties are given here. The number of lines used are given in parentheses. ${ }^{(a)}$ Solar abundances from Grevesse et al. (2007).

Table 5. Abundances of $s$-process elements.

\begin{tabular}{|c|c|c|c|c|c|c|c|}
\hline Star & {$[\mathrm{Sr} / \mathrm{Fe}]$} & {$[\mathrm{Y} / \mathrm{Fe}]$} & {$[\mathrm{Zr} / \mathrm{Fe}]$} & {$[\mathrm{Ba} / \mathrm{Fe}]$} & {$[\mathrm{La} / \mathrm{Fe}]$} & {$[\mathrm{Ce} / \mathrm{Fe}]$} & {$[\mathrm{s} / \mathrm{Fe}]$} \\
\hline V1261 Ori & - & $1.11 \pm 0.58$ & $56 \pm 0.54$ & 62 (3) & $1.44 \pm 0.54$ & $1.29 \pm 0.24$ & 1.3 \\
\hline IT Vir & $0.97 \pm 0.26$ & $0.94 \pm 0.30$ & $1.86 \pm 0.38$ & $2.33 \pm 0.35$ & $1.74 \pm 0.39$ & $1.27 \pm 0.37$ & .55 \\
\hline $56 \mathrm{Peg}$ & $0.58 \pm 0.16(2)$ & $0.46 \pm 0.28$ & $0.31 \pm 0.17$ & $1.46 \pm 0.28$ & $0.44 \pm 0.24$ & $0.16 \pm 0.24$ & \\
\hline DR Dra & 0.26 & $0.06 \pm 0.27$ & $0.33 \pm 0.16$ & $0.43 \pm 0.22$ & $0.18 \pm 0.23$ & $0.00 \pm 0.22$ & 0.2 \\
\hline$\zeta$ Cyg & 0.44 & $0.42 \pm 0.22$ & $0.39 \pm 0.24$ & $1.02 \pm 0.31$ & $0.40 \pm 0.22$ & $0.21 \pm 0.24(11)$ & \\
\hline IP Eri & (1) & $-0.24 \pm$ & $24 \quad(7)$ & $21 \quad(3)$ & $26(6)$ & $22(4)$ & 21 \\
\hline V1379 Aql & (1) & $0.25 \pm 0.18$ & $16(2)$ & $22(3)$ & $23(3)$ & $27 \quad(3)$ & 0. \\
\hline HR5692 & 1) & $25 \quad(6)$ & $23(11)$ & $21 \quad(5)$ & $3(11)$ & $24 \quad(9)$ & 0.4 \\
\hline$\zeta \mathrm{C}$ & (2) & $28 \quad(9)$ & $3(11)$ & $25 \quad(3)$ & $5(11)$ & $27(10)$ & 1.2 \\
\hline 63 Eri & (2) & $03 \pm 0.20(10)$ & $22(8)$ & 29 (4) & $21(10)$ & $21(9)$ & 0.18 \\
\hline 14 Aur C & $0.23 \pm 0.13$ & $-0.07 \pm 0.19$ & $0.17 \pm 0.15$ & $0.54 \pm 0.25$ & $0.20 \pm 0.23$ & $-0.09 \pm 0.29$ & $0.16 \pm 0.23$ \\
\hline
\end{tabular}

Notes. Total uncertainties are given here.

Table A.1), except in V1379 Aq1, V1261 Ori, and IT Vir, where they are not visible or too blended to be useful. This is why we prefer to use lines of diatomic carbon $\mathrm{C}_{2}$ to derive carbon abundances. Lines of $C_{2}$ appear roughly between 400 and $600 \mathrm{~nm}$. In the molecular line lists, we first identified useful lines of $\mathrm{C}_{2}$ by comparing synthetic spectra (for typical values of the atmospheric parameters) including $\mathrm{C}_{2}$ lines alone and including all lines to identify the least blended $\mathrm{C}_{2}$ lines. Then we compared these with observed spectra and compiled a selection of $\sim 20$ lines (Table B.1) that are visible in at least two-thirds of the objects. The line at $513.56 \mathrm{~nm}$ used by Barbuy et al. (1992) is also included. Good fits of $\mathrm{C}_{2}$ bands are difficult to achieve, but the lines are so sensitive to the carbon abundance that the latter can be easily inferred even from rough fits. In 14 Aur C, rotation broadens the lines so much (see Fig. 3) that only three lines can be used to derive the carbon abundance, even though they are blended with others. Especially the $\mathrm{C}_{2}$ line at $468.427 \mathrm{~nm}$ appears useful for this star (it is not used for the other stars because the line position in the synthetic spectrum appears to be somewhat redder than observed). The $\mathrm{C} / \mathrm{O}$ ratio was then calculated and is listed in Table 3.

We selected ten Ni lines (Table A.1). For the range of atmospheric parameters under consideration, $\mathrm{N}$ I lines are always very weak and blended with $\mathrm{CN}$ lines. The $\mathrm{N}$ abundance thus combines $\mathrm{NI}$ and $\mathrm{CN}$ lines, using the carbon abundance from the previous step. The $\mathrm{CN}$ lines are numerous and span the full HERMES spectral range. We selected 121 unblended or weakly blended CN lines in the range 640 to $890 \mathrm{~nm}$ (Table B.1). All $\mathrm{CN}$ lines were checked by eye because many telluric lines are present in this wavelength range and can be strongly blended with $\mathrm{CN}$ lines. Below $500 \mathrm{~nm}, \mathrm{CN}$ lines are too strongly blended by atomic and molecular features (mainly from $\mathrm{CH}, \mathrm{C}_{2}, \mathrm{MgH}$, and $\mathrm{SiH}$ ) to be of any use. NH lines cannot be used because they are located beyond the violet edge of the spectrograph $(370 \mathrm{~nm})$. For the fast rotator 14 Aur C, almost no $\mathrm{CN}$ lines can be used, since weak lines are blended by rotational broadening. We nevertheless used two of the selected CN lines and two other weak lines that were not in the original selection (at $\sim 871.15$ and $871.88 \mathrm{~nm}$ ). The $\mathrm{N}$ abundance in 14 Aur $\mathrm{C}$ is uncertain since the weak lines are at the limit of the spectral noise.

The S star V1261 Ori requires a specific way to derive its carbon abundance since atomic and $\mathrm{C}_{2}$ lines are immersed in $\mathrm{TiO}$ and $\mathrm{ZrO}$ bands. Infrared bands from $\mathrm{CO}, \mathrm{CN}$ and $\mathrm{OH}$ around $2 \mu \mathrm{m}$ must be used instead, as was done by Smith \& Lambert (1990) (with atmospheric parameters $T_{\text {eff }}=3650 \mathrm{~K}, \log g=$ $0.8,[\mathrm{Fe} / \mathrm{H}]=-0.52)$. We therefore used their $\mathrm{CNO}$ values to determine the subsequent $s$-process elements.

The derivation of CNO abundances in IT Vir is particularly challenging and consistently leads to a $[\mathrm{N} / \mathrm{Fe}]$ ratio above 1.0 dex. We proceeded as follows: we first estimated the $\mathrm{O}$ abundance through the [O I] $630.0 \mathrm{~nm}$ line using spectral syntheses computed from dedicated S-star model atmospheres with the parameters listed in Table 2 and three different values of the $\mathrm{C} / \mathrm{O}$ ratio $(0.50,0.75$ and 0.90$)$. Basically, as the $\mathrm{C} / \mathrm{O}$ ratio increases (for a given $\mathrm{O}$ abundance), the strength of the forbidden line decreases. This strength is well reproduced for $[\mathrm{O} / \mathrm{Fe}]=$ $+0.20,+0.40$, and +0.60 dex. Then, for each $\mathrm{C} / \mathrm{O}$ ratio and the corresponding $\mathrm{O}$ abundance, a carbon abundance ensues. 
We determined which of these carbon abundances best fit the $\mathrm{C}_{2}$ lines (between 460 and $560 \mathrm{~nm}$ ), but we could only exclude the value corresponding to $\mathrm{C} / \mathrm{O}=0.5$; the other two possibilities led to similarly good fits, they could not be distinguished. From the above analysis, we can at least be confident that the $\mathrm{C} / \mathrm{O}$ ratio is intermediate between the solar value and unity, which disqualifies the SC spectral type quoted in the literature. For each remaining pair of $(\mathrm{C} / \mathrm{O},[\mathrm{O} / \mathrm{Fe}])$ values, we finally computed synthetic spectra for three different $[\mathrm{N} / \mathrm{Fe}]$ values $(0.0$, +1.0 and +1.5 dex $)$ and evaluated how well these synthetic spectra fit the observed spectrum in the three wavelength ranges [629-631], [776.5-778.5], and [843.5-845.5] $\mathrm{nm}$ that contain $\mathrm{CN}$ lines. Good fits are obtained for $[\mathrm{N} / \mathrm{Fe}]=1.5 \mathrm{dex}$ when $\mathrm{C} / \mathrm{O}=0.75,[\mathrm{O} / \mathrm{Fe}]=0.40 \mathrm{dex}$ and for $[\mathrm{N} / \mathrm{Fe}]=1.0 \mathrm{dex}$ when $\mathrm{C} / \mathrm{O}=0.90,[\mathrm{O} / \mathrm{Fe}]=0.60$. Again, we cannot distinguish between these two equally good fits. Therefore both possibilities are listed in Table 3. Nevertheless, whenever necessary in the remainder of the study, we adopt the former set.

We checked the sensitivity to gravity $\log g$ of our conclusion of a large $\mathrm{N}$ overabundance. Adopting $\log g=0$ instead of 1 as above, all other parameters being equal (with $\mathrm{C} / \mathrm{O}=0.75$ ), we obtain a value of $[\mathrm{N} / \mathrm{Fe}]$ still higher than 1.0. Similarly, increasing the temperature does not permit lowering the $[\mathrm{N} / \mathrm{Fe}]$ value below 1.0 dex. This result is therefore probably robust.

\section{2. s-process abundances}

Atomic lines were carefully selected over the whole HERMES spectral range using both synthetic and observed spectra. They are listed in Table A.1. Whenever possible, we tried to select lines for each species from both neutral and singly ionized states (excluding strong resonance lines of neutral species known to suffer from NLTE effects).

The only observable lines of Sr II are the strong lines below $430.5 \mathrm{~nm}$, which are too strongly blended to derive reliable abundances. We therefore only used Sr I lines. Of the eight selected Sr I lines, the line at $687.83 \mathrm{~nm}$ is often blended by telluric $\mathrm{O}_{2}$, the resonance line at $460.73 \mathrm{~nm}$ is known to suffer from NLTE effects and to give abundances lower than that from higher excitation lines. The line at $707.01 \mathrm{~nm}$ has been used in all the target stars except for 14 Aur C and V1261 Aur. In 14 Aur C, the two lines at 640.85 and $707.01 \mathrm{~nm}$ are not observable because they blend with other lines as a result of rapid rotation or are lost in the noise. Instead, the strong Sr II line around $416 \mathrm{~nm}$ seems to provide a reliable estimate of the $\mathrm{Sr}$ abundance. In V1261 Ori, the two Sr I lines at 640.85 and $707.01 \mathrm{~nm}$ are too blended, and we used instead two SrI lines at 687.83 and $689.26 \mathrm{~nm}$, even though the continuum placement remains the largest uncertainty.

For Y, we mainly used clean lines of Y II that are more numerous than Y I lines. The cleanest and most useful Y II lines in our sample are located at 528.98, 540.28, 572.89, and $679.54 \mathrm{~nm}$. The other lines used are listed in Appendix A.

For Zr, we mainly used lines of Zr I, since they are more numerous than Zr II lines. Lines of Zr I between 800 and $850 \mathrm{~nm}$, when useful, give abundances systematically stronger by about $\sim 0.5$ dex. For 14 Aur C, only Zr II lines below $540 \mathrm{~nm}$ can be used for the abundance determination. Since V1261 Ori is veiled by $\mathrm{ZrO}$ molecular bands, we used the same lines (between 740 and $758 \mathrm{~nm}$ ) as those used by Smith \& Lambert (1990) to derive the $\mathrm{Zr}$ abundance. Lines above $800 \mathrm{~nm}$ are useable but give somewhat higher abundances, as already mentioned.

For $s$-process elements belonging to the second peak ( $\mathrm{Ba}, \mathrm{La}$, and $\mathrm{Ce}$ ), only neutral lines are available for $\mathrm{Ba}$. We did not use the Ba II resonance lines at 455.4 and $493.4 \mathrm{~nm}$ in the abundance analysis because of the known NLTE effects (see e.g. Olshevsky \& Shchukina 2007), except in 14 Aur C, where too few other lines are available. We mainly used the Ba II lines at 585.37, 614.17, and $649.69 \mathrm{~nm}$ and the Ba I line at $748.81 \mathrm{~nm}$.

Many lines (about 30) are available for $\mathrm{La}$ and $\mathrm{Ce}$ in the HERMES wavelength range. The most useful La II lines are located at 529.08, 530.20, 530.35, 617.27, 639.05, and $677.43 \mathrm{~nm}$. The most useful Ce II lines are located at 456.24, 533.06, 604.34, $802.56,871.67$, and $877.21 \mathrm{~nm}$. The Ce II lines are the strongest in V1261 Ori, which indeed has the largest Ce overabundance $([\mathrm{Ce} / \mathrm{Fe}]=1.29 \mathrm{dex})$ in our sample.

To illustrate the good agreement between observed and synthetic spectra, we show in Fig. 3 the spectral region around the Ba II $585.36 \mathrm{~nm}$ line, which is well visible in the entire sample. The synthetic spectra in blue are computed with $[\mathrm{Ba} / \mathrm{Fe}]=0$, whereas the red ones are computed with the appropriate $[\mathrm{Ba} / \mathrm{Fe}]$ abundances as listed in Table 5. The red synthetic spectra nicely reproduce the observed spectra, especially for the fast rotators V1379 Aql and 14 Aur C. The fit is slightly worse for the cooler S stars V1261 Ori and IT Vir because of the presence of numerous molecular lines, but it is nevertheless possible to derive the Ba abundance reliably.

\subsection{Uncertainties on s-process abundances}

We investigated the effect on the $s$-process abundances of the uncertainties from the atmospheric parameters, adopting as typical uncertainties on these parameters (Table 2) the values $\sigma_{T_{\text {eff }}}= \pm 50 \mathrm{~K}, \sigma_{\log g}= \pm 0.3 \mathrm{dex}, \sigma_{[\mathrm{Fe} / \mathrm{H}]}= \pm 0.10 \mathrm{dex}$, and $\sigma_{\xi}= \pm 0.10 \mathrm{~km} \mathrm{~s}^{-1}$.

The statistical uncertainty $\sigma_{\text {stat }}$ listed in Table 6 is the lineto-line dispersion divided by $\sqrt{N}$, where $N$ is the number of lines used for a given element (Table 6). This statistical uncertainty takes not only uncertainties on atomic data into account, but also those on continuum location and line-profile mismatch problems.

Statistical $\left(\sigma_{\text {stat }}\right)$ and model-induced (i.e. $\sigma_{T_{\text {eff }}}, \sigma_{\log g}, \sigma_{[\mathrm{Fe} / \mathrm{H}]}$, and $\sigma_{\xi}$ ) uncertainties were quadratically added (thus assuming independence between them, as is common practice, although in reality they may be correlated, as discussed by Johnson 2002) to obtain the total uncertainty $\sigma_{\text {tot }}$ for each $s$-process element (which may thus be overestimated). We performed this analysis on $\zeta$ Cyg as it is representative of the whole sample (see Fig. 4). The estimated uncertainties are given in Table 6. We note that the uncertainties on the absolute $s$-process abundances given in Table 4 are only the line-by-line dispersion.

To derive the uncertainties on the various $[\mathrm{X} / \mathrm{Fe}]$ abundances (as listed in Table 5), we added the uncertainties on the absolute abundances $A(\mathrm{Fe})$ and $A(\mathrm{X})$ quadratically. The resulting uncertainties are expressed as

$\sigma_{\mathrm{X}}=\sqrt{\sigma_{\text {tot }}(\mathrm{X})^{2}+\sigma(\mathrm{Fe})^{2}+\sigma_{\odot}(\mathrm{X})^{2}+\sigma_{\odot}(\mathrm{Fe})^{2}}$,

where $\sigma_{\odot}(\mathrm{X})$ denotes the uncertainty on the solar abundance of species $X$ as given by Grevesse et al. (2007). The corresponding $\sigma_{\mathrm{X}}$ uncertainties are listed in Table 5 and plotted in Figs. 4 and 5.

\subsection{Comparison with previous studies}

V1261 Ori (HD 35155). This star has been analysed by Smith \& Lambert (1990), who found the second-peak $s$-process elements $(\mathrm{Ba}, \mathrm{La}, \mathrm{Ce}, \mathrm{Nd})$ to be enriched by more than $1 \mathrm{dex}$, as we do. However, Smith \& Lambert (1990) found no overabundance in excess of 0.23 dex for $\mathrm{Y}$ and $\mathrm{Zr}$, whereas we find these 


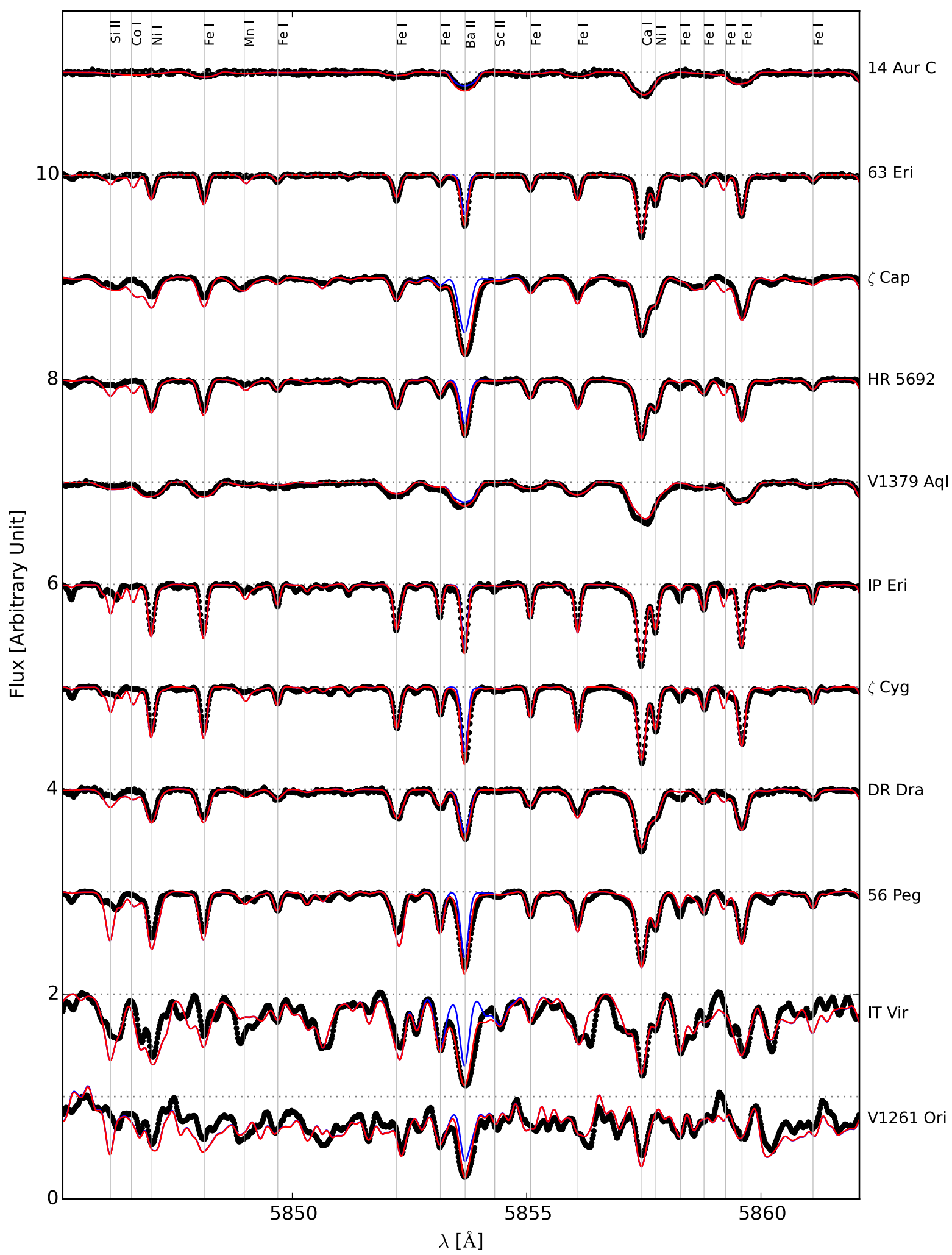

Fig. 3. Example of the observed and synthetic spectra around the Ba II line at $585.36 \mathrm{~nm}$. The solid blue line corresponds to $[\mathrm{Ba} / \mathrm{Fe}]=0$.

elements to be overabundant by more than 1 dex. We stress that both the models and the line data are very different between the two studies.

IT Vir. Smith (1984) derived the CNO abundances in IT Vir, but did not confirm the large $\mathrm{N}$ abundance that we obtain for that $\operatorname{star}([\mathrm{N} / \mathrm{Fe}]=1.1$ to $1.4 \mathrm{dex}-$ Table $3-$, as compared to
0.52 dex for Smith 1984). This large N overabundance is accompanied with a low ${ }^{12} \mathrm{C} /{ }^{13} \mathrm{C}$ ratio of 8 (Smith 1984), which could hint at $\mathrm{CN}$ processing. However, similarly low ${ }^{12} \mathrm{C} /{ }^{13} \mathrm{C}$ ratios are a common feature among barium stars (Smith 1984; Barbuy et al. 1992), without being associated with $\mathrm{N}$ overabundances as large as the one we found for IT Vir. We found that 
Table 6. Uncertainties on the derived abundances in $\zeta$ Cyg.

\begin{tabular}{lrllcccc|c}
\hline \hline $\mathrm{X}$ & $N$ & $\sigma_{T_{\text {eff }}}$ & $\sigma_{\log g}$ & $\sigma_{[\mathrm{Fe} / \mathrm{H}]}$ & $\sigma_{\xi}$ & $\sigma_{\text {atm }}$ & $\sigma_{\text {stat }}$ & $\sigma_{\text {tot }}$ \\
\hline $\mathrm{Sr}$ & 1 & 0.05 & 0.01 & 0.01 & 0.01 & 0.05 & - & 0.05 \\
$\mathrm{Y}$ & 7 & 0.03 & 0.10 & 0.01 & 0.04 & 0.11 & 0.06 & 0.13 \\
$\mathrm{Zr}$ & 9 & 0.09 & 0.01 & 0.01 & 0.01 & 0.09 & 0.07 & 0.12 \\
$\mathrm{Ba}$ & 4 & 0.09 & 0.09 & 0.06 & 0.05 & 0.15 & 0.11 & 0.19 \\
$\mathrm{La}$ & 9 & 0.03 & 0.16 & 0.03 & 0.01 & 0.17 & 0.03 & 0.17 \\
$\mathrm{Ce}$ & 11 & 0.03 & 0.14 & 0.05 & 0.02 & 0.15 & 0.04 & 0.16 \\
\hline
\end{tabular}

Notes. $\sigma_{i}$ are the uncertainties caused by the errors on the atmospheric parameters $\left(i=T_{\text {eff }}, \log g,[\mathrm{Fe} / \mathrm{H}], \xi\right.$; see text) and $\sigma_{\text {stat }}$ is the line-toline scatter on the abundance divided by $\sqrt{N}$, where $N$ is the number of lines used. The individual model-driven uncertainties are quadratically added to yield the total model-driven uncertainty $\sigma_{\text {atm }}$, which in turn is quadratically added to the statistical uncertainty to give the total uncertainty $\sigma_{\text {tot }}$.

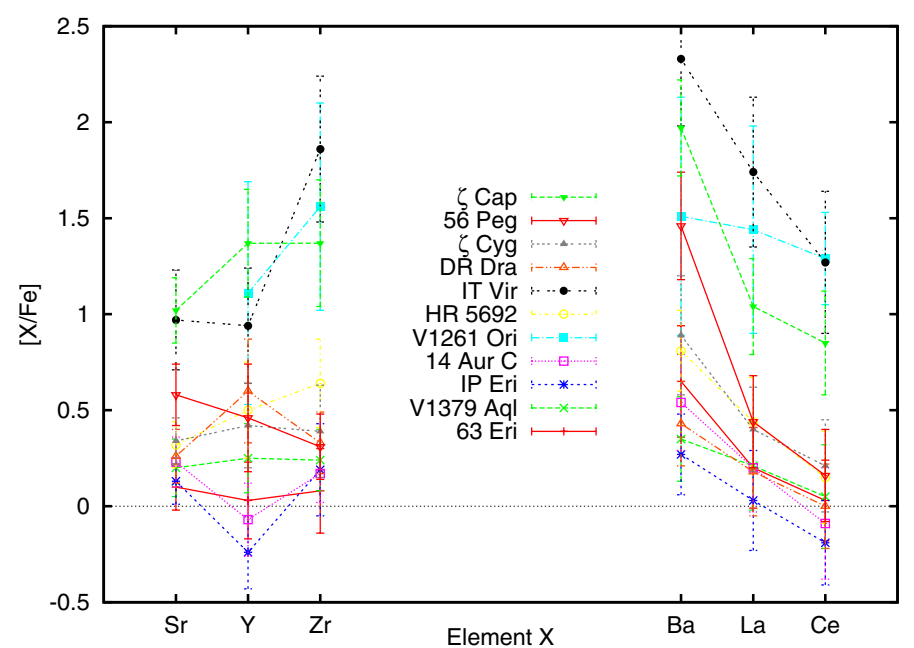

Fig. 4. First-peak (Sr, Y, Zr) and second-peak (Ba, La, Ce) s-process abundances relative to $\mathrm{Fe}$.

regardless of the effective temperature or gravity adopted for this $\operatorname{star}\left(T_{\text {eff }}=3900\right.$ or $4200 \mathrm{~K}, \log g=0$ or 1 dex; see Sect. 4$)$, the large $[\mathrm{N} / \mathrm{Fe}]$ overabundance remains. Even after carefully rederiving $\mathrm{O}$ and $\mathrm{C}$ abundances, $[\mathrm{N} / \mathrm{Fe}]$ is still larger than 1 dex. Formerly, the largest $\mathrm{N}$ abundances observed in barium stars were $[\mathrm{N} / \mathrm{Fe}]=0.7 \mathrm{dex}$ in HD 27271 and 0.65 dex in HD 60197 (Barbuy et al. 1992). It may be relevant here to note that IT Vir has one of the largest Roche-lobe filling factors of barium stars, since it has a moderately short orbital period (186 d), but a comparatively large radius $\left(\sim 55 R_{\odot}\right.$; Jorissen et al. 1995). As a consequence, Jorissen and collaborators reported ellipsoidal variations for this star. The associated faster rotation, tidal deformations, and departure from spherical symmetry will trigger meridional currents and deep mixing, and the observed large $\mathrm{N}$ abundance in IT Vir could perhaps be related to such processes currently occurring in the red giant (see for instance Decressin et al. 2009, for a discussion of rotation-induced mixing and the resulting surface enhancement in nitrogen).

The $s$-process abundances agree well for $\mathrm{Sr}, \mathrm{Y}$, and $\mathrm{Ce}$, but $\mathrm{Zr}, \mathrm{Ba}$, and $\mathrm{La}$ are found to be more abundant by about 1 dex in our study than the values reported by Smith (1984).

$56 \mathrm{Peg}$. It is the first time that an $s$-process abundance analysis is performed for $56 \mathrm{Peg}$, and it confirms its barium nature. Because of the high luminosity of $56 \mathrm{Peg}$ (it is classified as K0Iab confirmed by the low gravity of $\log g=1.3$; Table 2 ), the increased strength of the Ba II lines, which led to its classification as a barium star, might have been caused by the low atmospheric pressure, a consequence of Saha equation. But our abundance analysis reveals a weak albeit clear $s$-process enhancement.

$\zeta$ Cap and $\zeta$ Cyg. These stars were analysed by Smiljanic et al. (2007). The agreement between the two studies on the $s$-process abundances is reasonable (in particular, both studies find large overabundances in $\zeta$ Cap and much milder ones in $\zeta$ Cyg), except for $\mathrm{Sr}$ in $\zeta$ Cap: Smiljanic et al. (2007) obtained $[\mathrm{Sr} / \mathrm{Fe}]=2.21$, as compared to 1.02 in the present study. Smiljanic et al. (2007) used the single Sr I resonance line at $460.73 \mathrm{~nm}$, whereas we used that line and a line at $707.01 \mathrm{~nm}$. Based solely on the $460.73 \mathrm{~nm}$ line, we find $[\mathrm{Sr} / \mathrm{Fe}]=0.99$, and this value agrees well with the value 1.07 based on the $707.01 \mathrm{~nm}$ line. The reason for the discrepancy between the $\mathrm{Sr}$ abundances derived from the $\mathrm{Sr}$ I resonance line at $460.73 \mathrm{~nm}$ here and in Smiljanic et al. (2007) must partly reside in the different $\log g f$ values used [ 0.069 by Smiljanic et al. (2007) against 0.283 in our work, from NIST with the AA accuracy - see Appendix A], and partly in the different atmospheric parameters adopted.

DR Dra. Berdyugina (1994), Zacs et al. (1997), and Barisevičius et al. (2010) concluded, like we do, that DR Dra does not exhibit any $s$-process overabundance.

IP Eri. This star has been analysed by Merle et al. (2014). We rederived the $s$-process abundances with the same pipeline and methodology, but with a refined line selection for each element. The rederived $s$-process abundances agree with our previous work within the error bars, and we here obtain lower statistical uncertainties.

\section{Discussion}

The s-process abundance distribution is presented in Fig. 4, which clearly shows that our sample contains both normal stars and stars enriched in $s$-process elements (and the latter also exhibit enhanced carbon and sometimes nitrogen overabundances; Fig. 6). The origin of the difference between these two stellar categories is clearly revealed when plotting the average $s$-process abundance, as listed in the last column of Table 5, as a function of the mass of the WD companion (Fig. 5). The expected trend is indeed obtained: $s$-process overabundances are found in binary stars with a companion WD mass higher than about $0.5 M_{\odot}$, that is, the progenitor of the WD went through the thermally pulsing AGB (TP-AGB) phase, was able to synthesize $s$-process elements in the intershell zone, and these were subsequently dredged up to the surface. Indeed, Hurley et al. (2000) (their Eq. (66)) predicted the minimum CO core mass at the base of the AGB (just at the end of core He-burning) to be at least $0.51 M_{\odot}$ (for a star of initial mass $0.9 M_{\odot}$ ). Conversely, when the WD mass is lower than $0.51 M_{\odot}$, the $s$-process synthesis did not occur (because the progenitor did not reach the TP-AGB): $s$-process overabundances are not observed. There are two marginal cases, namely DR Dra and 14 Aur C, whose WD mass qualifies them for being polluted by $s$-process material, but which are not. For 14 Aur C, apart from the difficulty imprinted on the analysis by the rapid stellar rotation, the cause for the absence of $s$-process enrichment could be its unusually short period of three days, which is likely the result of a common-envelope evolution (Han et al. 1995). It is believed that this channel does not lead to substantial accretion during the common-envelope process (Ricker $\&$ Taam 2008) ${ }^{2}$. Nevertheless, the recent occurrence of binary

2 The low-metallicity carbon dwarf HE 0024-2523, with its $3.4 \mathrm{~d}$ orbital period and its strong $\mathrm{Pb}$ overabundance, is a noticeable counterexample of this statement, however (Lucatello et al. 2003). 
T. Merle et al.: To Ba or not to Ba?
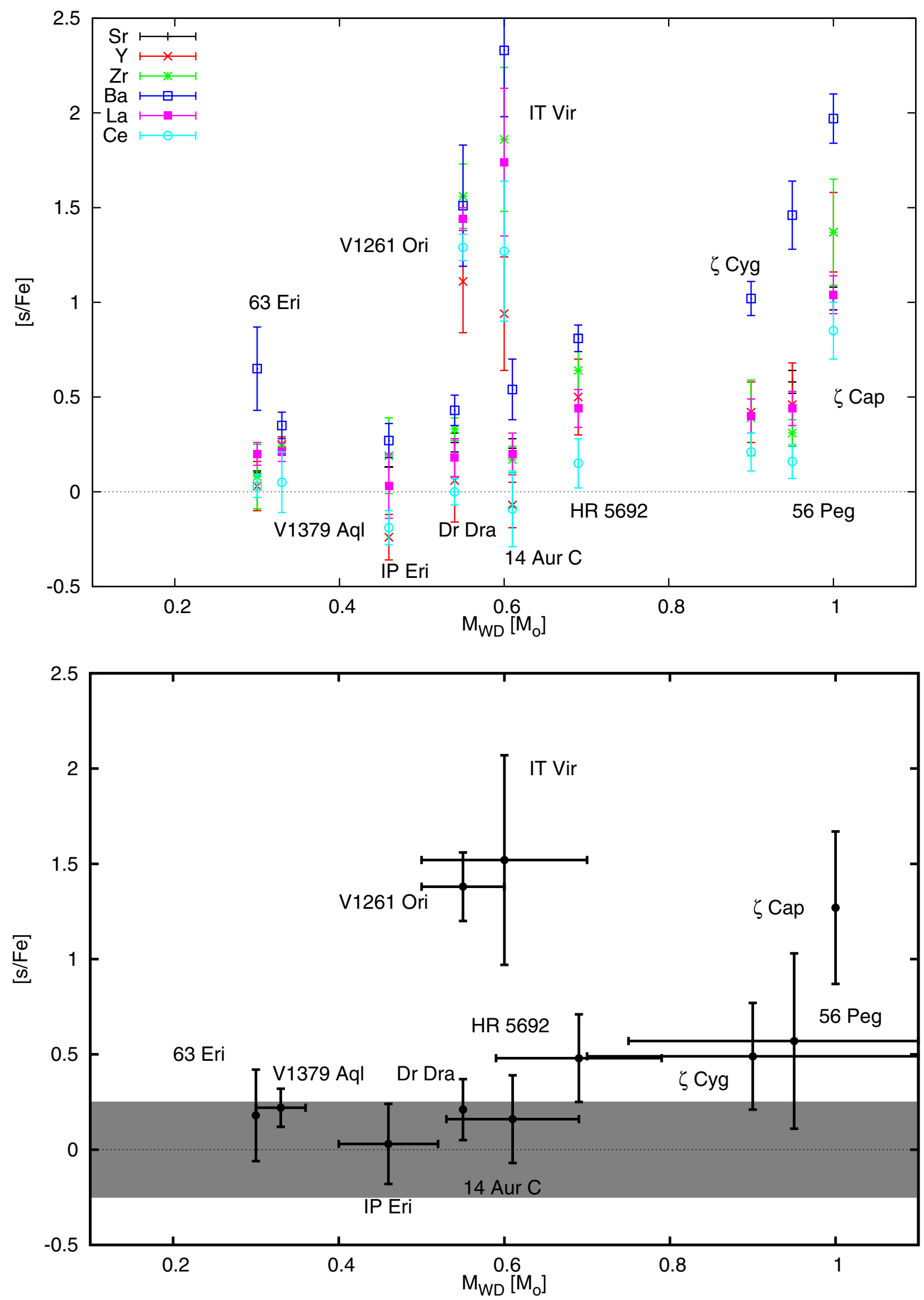

Fig. 5. Upper panel: first- (Sr, Y, Zr) and second-peak (Ba, La, Ce) s-process abundances relative to Fe as a function of the WD mass. Lower panel: same as upper panel, but with mean $s$-process abundances relative to Fe. To guide the eye, the grey zone shows the \pm 0.25 dex zone without significant enhancement in $s$-process abundances. 


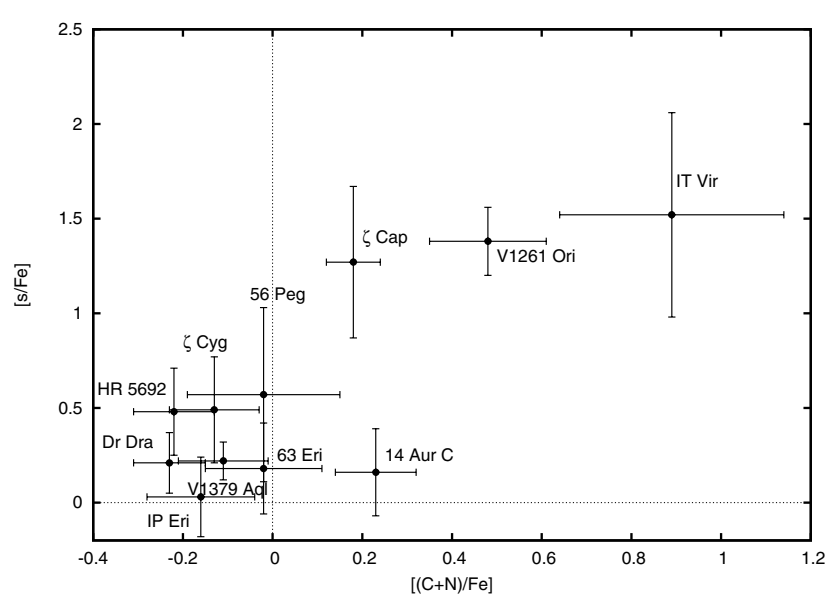

Fig. 6. Correlation between the $s$-process abundances and the carbon + nitrogen abundances.

interaction is revealed by the rapid rotation of the star. The same holds true for DR Dra, whose absence of $s$-process overabundance is slightly more puzzling, however. The long period of DR Dra (904 d) suggests that the rapid rotation responsible for the chromospheric activity be due to spin accretion (Theuns et al. 1996) rather than to tidal coupling, which requires a closer system (Fekel et al. 1993). Thus there has been some binary interaction in the recent past of DR Dra. The absence of $s$-process overabundance in this star suggests that with a mass estimated at $0.55 M_{\odot}$ (Fekel \& Simon 1985), either the WD does not originate from a TP-AGB star (Hurley et al. 2000), there was no dredge-up in the TP-AGB star, or there was simply no $s$-process nucleosynthesis operating.

The level of $s$-process overabundances of barium stars varies greatly, from the marginal level of HR 5692, $\zeta$ Cyg, and $56 \mathrm{Peg}$, to the highest level of IT Vir. Two co-existing causes for these variations are readily identified in the literature: on the one hand, the efficiency of the $s$-process nucleosynthesis in AGB stars varies with metallicity (Clayton 1988; Käppeler et al. 2011), and on the other hand, the efficiency of mass accretion varies with orbital separation (Jorissen \& Boffin 1992; Han et al. 1995; Pols et al. 2003). Thus, orbital separation and metallicity could directly control the level of $s$-process overabundances in barium stars. But all previous studies have concluded that if it exists at all, the correlation between orbital periods and $s$-process overabundances is weak, and the present study is no exception. For instance, 56 Peg has one of the shortest orbital periods ${ }^{3}$ of all barium stars (111 d; Griffin 2006), and yet its $s$-process overabundance level is very moderate. A search for additional parameters (besides metallicity) that might blur the relationship between orbital periods and $s$-process pollution levels is therefore of interest. Here we suggest that the WD mass, tracing the maximum luminosity reached on the AGB (through the core-mass luminosity relationship; Herwig et al. 1998), could also play a role in this respect. Indeed, the AGB luminosity is a proxy for the number of thermal pulses experienced by the AGB star (although the initial AGB mass must play a role as well), which in turn governs the $s$-process level attained in its atmosphere. However, too few stars are available at present to be able to draw any firm conclusion, apart from the observation that large $s$-process overabundance levels are found at both ends of the CO

\footnotetext{
3 We implicitly assume here that the range of system masses is moderate enough for orbital periods to be a good proxy for the orbital separations.
}

WD mass range: around $0.6 M_{\odot}$ (IT Vir, V1261 Ori) and around $1 M_{\odot}(\zeta$ Cap $)$.

\section{Conclusion}

We have analysed a sample of 11 binary systems comprising WD of known masses. High-resolution and high signal-to-noise spectra were collected from the HERMES spectrograph installed at the Mercator $1.2 \mathrm{~m}$ telescope. The atmospheric parameters and the CNO and $s$-process abundances were derived using LTE models. We derived for the first time atmospheric parameters for 14 Aur C (HD 33959C) and s-process element abundances for 56 Peg (HR 8796). The sample includes two $S$ stars that were analysed with new dedicated S-star MARCS model atmospheres. One of them, IT Vir (HD 121447), has the largest overabundance of $s$-process elements in the sample and has been identified as highly N-enriched. Such a high level of $\mathrm{N}$ overabundance could perhaps be related to the photometric variations exhibited by this star and is attributed to ellipsoidal variations as the red giant has a large Roche-filling factor. The ensuing faster rotation could trigger deep mixing, which is known to bring nitrogen to the surface.

Finally, from our homogeneous study of $s$-process abundances in the primary components of binary systems involving a WD companion, we find that there is a clear relationship between $s$-process enrichment and WD mass: as expected, primary components in binary systems involving He WDs (thus with a mass lower than about $0.5 M_{\odot}$ ) never show $s$-process overabundances, as the WD progenitor never reached the TP-AGB.

Acknowledgements. This research has been funded by the Belgian Science Policy Office under contract BR/143/A2/STARLAB. T.M. is supported by the FNRS-F.R.S. as temporary post-doctoral researcher under grant No. 2.4513.11. This work was supported by the Fonds de la Recherche Scientifique FNRS under Grant no T.0198.13. The Mercator telescope is operated thanks to grant number G.0C31.13 of the FWO under the "Big Science" initiative of the Flemish governement. Based on observations obtained with the HERMES spectrograph, supported by the Fund for Scientific Research of Flanders (FWO), the Research Council of K.U.Leuven, the Fonds National de la Recherche Scientifique (F.R.S.FNRS), Belgium, the Royal Observatory of Belgium, the Observatoire de Genève, Switzerland and the Thüringer Landessternwarte Tautenburg, Germany. This work has made use of the VALD database, operated at Uppsala University, the Institute of Astronomy RAS in Moscow, and the University of Vienna.

\section{References}

Abia, C., \& Wallerstein, G. 1998, MNRAS, 293, 89

Ake, T. B. 1979, ApJ, 234, 538

Ake, III, T. B., Johnson, H. R., \& Ameen, M. M. 1991, ApJ, 383, 842

Alvarez, R., \& Plez, B. 1998, A\&A, 330, 1109

Asplund, M. 2005, ARA\&A, 43, 481

Böhm-Vitense, E., Carpenter, K., Robinson, R., Ake, T., \& Brown, J. 2000, ApJ, 533, 969

Barbuy, B., Jorissen, A., Rossi, S. C. F., \& Arnould, M. 1992, A\&A, 262, 216

Barisevičius, G., Tautvaišienė, G., Berdyugina, S., Chorniy, Y., \& Ilyin, I. 2010, Balt. Astron., 19, 157

Berdyugina, S. V. 1994, Astron. Lett., 20, 796

Bessell, M. S., Castelli, F., \& Plez, B. 1998, A\&A, 333, 231

Bidelman, W. P., \& Keenan, P. C. 1951, ApJ, 114, 473

Bilíková, J., Chu, Y.-H., Gruendl, R. A., \& Maddox, L. A. 2010, AJ, 140, 1433

Böhm-Vitense, E. 1980, ApJ, 239, L79

Böhm-Vitense, E., Nemec, J., \& Proffitt, C. 1984, ApJ, 278, 726

Burleigh, M. R., Barstow, M. A., \& Fleming, T. A. 1997, MNRAS, 287, 381

Burleigh, M. R., Barstow, M. A., \& Holberg, J. B. 1998, MNRAS, 300, 511

Christian, D. J., Vennes, S., Thorstensen, J. R., \& Mathioudakis, M. 1996, AJ, 112,258

Clayton, D. D. 1988, MNRAS, 234, 1

Decressin, T., Charbonnel, C., Siess, L., et al. 2009, A\&A, 505, 727

Den Hartog, E. A., Lawler, J. E., \& Wood, M. P. 2014a, ApJS, 215, 7

Den Hartog, E. A., Ruffoni, M. P., Lawler, J. E., et al. 2014b, ApJS, 215, 23 
Dominy, J. F. \& Lambert, D. L. 1983, ApJ, 270, 180

Fekel, F. X., \& Simon, T. 1985, AJ, 90, 812

Fekel, F. C., Henry, G. W., Busby, M. R., \& Eitter, J. J. 1993, AJ, 106, 2370

Frankowski, A., \& Jorissen, A. 2006, The Observatory, 126, 25

Gray, R. O., Corbally, C. J., Garrison, R. F., McFadden, M. T., \& Robinson, P. E. 2003, AJ, 126, 2048

Gray, R. O., McGahee, C. E., Griffin, R. E. M., \& Corbally, C. J. 2011, AJ, 141, 160

Grevesse, N., Asplund, M., \& Sauval, A. J. 2007, Space Sci. Rev., 130, 105

Griffin, R. F. 2006, The Observatory, 126, 1

Griffin, R. F., \& Keenan, P. C. 1992, The Observatory, 112, 168

Gustafsson, B., Edvardsson, B., Eriksson, K., et al. 2008, A\&A, 486, 951

Han, Z., Eggleton, P. P., Podsiadlowski, P., \& Tout, C. A. 1995, MNRAS, 277, 1443

Herwig, F., Schoenberner, D., \& Bloecker, T. 1998, A\&A, 340, L43

Hoard, D. W., Wachter, S., Sturch, L. K., et al. 2007, AJ, 134, 26

Holweger, H. 1979, in Liege International Astrophysical Colloquia 22, eds.

A. Boury, N. Grevesse, \& L. Remy-Battiau, 117

Hurley, J. R., Pols, O. R., \& Tout, C. A. 2000, MNRAS, 315, 543

Jeffries, R. D., \& Smalley, B. 1996, A\&A, 315, L19

Jofre, P., Heiter, U., Soubiran, C., et al. 2014, A\&A, 564, A133

Johnson, J. A. 2002, ApJS, 139, 219

Jorissen, A., \& Boffin, H. M. J. 1992, in Binaries as Tracers of Stellar Formation, eds. A. Duquennoy, \& M. Mayor (Cambridge: Cambridge University Press), 110

Jorissen, A., Mayor, M., Manfroid, J., \& Sterken, C. 1992, Information Bulletin on Variable Stars, 3730, 1

Jorissen, A., Hennen, O., Mayor, M., Bruch, A., \& Sterken, C. 1995, A\&A, 301, 707

Jorissen, A., Schmitt, J. H. M. M., Carquillat, J. M., Ginestet, N., \& Bickert, K. F. 1996, A\&A, 306, 467

Jorissen, A., Van Eck, S., Mayor, M., \& Udry, S. 1998, A\&A, 332, 877

Käppeler, F., Gallino, R., Bisterzo, S., \& Aoki, W. 2011, Rev. Mod. Phys., 83, 157

Kovacs, N. 1983, A\&A, 120, 21

Kupka, F., Dubernet, M.-L., \& VAMDC Collaboration. 2011, Balt. Astron., 20, 503

Landsman, W., Simon, T., \& Bergeron, P. 1993, PASP, 105, 841
Lucatello, S., Gratton, R., Cohen, J. G., et al. 2003, AJ, 125, 875

Masseron, T. 2006, Ph.D. Thesis, Observatoire de Paris, France

Masseron, T., Plez, B., Van Eck, S., et al. 2014, A\&A, 571, A47

McClure, R. D., \& Woodsworth, A. W. 1990, ApJ, 352, 709

McClure, R. D., Fletcher, J. M., \& Nemec, J. M. 1980, ApJ, 238, L35

Merle, T., Jorissen, A., Masseron, T., et al. 2014, A\&A, 567, A30

Neyskens, P., van Eck, S., Jorissen, A., et al. 2015, Nature, 517, 174

Olshevsky, V. L., \& Shchukina, N. G. 2007, in Modern solar facilities - advanced solar science, eds. F. Kneer, K. G. Puschmann, \& A. D. Wittmann (Göttingen: Universitäts Verlag), 307

Plez, B. 2012, Astrophysics Source Code Library, 5004

Pols, O. R., Karakas, A. I., Lattanzio, J. C., \& Tout, C. A. 2003, in Symbiotic Stars Probing Stellar Evolution, eds. R. L. M. Corradi, J. Mikolajewska, \& T. J. Mahoney, ASP Conf. Ser., 303, 290

Prugniel, P., Vauglin, I., \& Koleva, M. 2011, A\&A, 531, A165

Raskin, G., van Winckel, H., Hensberge, H., et al. 2011, A\&A, 526, A69

Ricker, P. M., \& Taam, R. E. 2008, ApJ, 672, L41

Ruffoni, M. P., Den Hartog, E. A., Lawler, J. E., et al. 2014, MNRAS, 441, 3127

Schindler, M., Stencel, R. E., Linsky, J. L., Basri, G. S., \& Helfand, D. J. 1982, ApJ, 263, 269

Siess, L., Davis, P. J., \& Jorissen, A. 2014, A\&A, 565, A57

Smiljanic, R., Porto de Mello, G. F., \& da Silva, L. 2007, A\&A, 468, 679

Smith, V. V. 1984, A\&A, 132, 326

Smith, V. V., \& Lambert, D. L. 1990, ApJS, 72, 387

Sneden, C., Lucatello, S., Ram, R. S., Brooke, J. S. A., \& Bernath, P. 2014, ApJS, 214, 26

Stefanik, R. P., Torres, G., Latham, D. W., et al. 2011, AJ, 141, 144

Theuns, T., Boffin, H. M. J., \& Jorissen, A. 1996, MNRAS, 280, 1264

Van Eck, S., Neyskens, P., Plez, B., et al. 2011, J. Phys. Conf. Ser., 328, 012009

van Leeuwen, F. 2007, A\&A, 474, 653

Vennes, S., Mathioudakis, M., Doyle, J. G., Thorstensen, J. R., \& Byrne, P. B. 1995, A\&A, 299, L29

Vennes, S., Christian, D. J., Mathioudakis, M., \& Doyle, J. G. 1997, A\&A, 318, L9

Vennes, S., Christian, D. J., \& Thorstensen, J. R. 1998, ApJ, 502, 763

Zacs, L., Musaev, F. A., Bikmaev, I. F., \& Alksnis, O. 1997, A\&AS, 122, 31 


\section{Appendix A: Atomic linelist}

Table A.1. Atomic line list.

\begin{tabular}{|c|c|c|c|}
\hline$\lambda[\mathrm{nm}]$ & $\chi[\mathrm{eV}]$ & $\log g f$ & \\
\hline C I & \multicolumn{2}{|c|}{$A_{\odot}(\mathrm{C})=8.39$} & \\
\hline 538.0337 & 7.685 & -1.615 & \\
\hline 555.3174 & 8.643 & -2.370 & \\
\hline 768.5190 & 8.771 & -1.519 & \\
\hline 833.5148 & 7.685 & -0.420 & \\
\hline 872.7126 & 1.264 & -8.136 & \\
\hline O I & \multicolumn{2}{|c|}{$A_{\odot}(\mathrm{O})=8.66$} & \\
\hline 615.6776 & 10.741 & -0.694 & \\
\hline 630.0304 & 0.000 & -9.715 & \\
\hline 636.3776 & 0.020 & -10.190 & \\
\hline 777.1941 & 9.146 & 0.369 & only in IT Vir \\
\hline 777.4161 & 9.146 & 0.223 & only in IT Vir \\
\hline 777.5388 & 9.146 & 0.001 & only in IT Vir \\
\hline 844.6359 & 9.521 & 0.236 & \\
\hline 844.6758 & 9.521 & 0.014 & \\
\hline Sr I & \multicolumn{2}{|c|}{$A_{\odot}(\mathrm{Sr})=2.92$} & \\
\hline 460.7327 & 0.000 & 0.283 & NIST AA \\
\hline 640.8459 & 2.271 & 0.510 & in the red wing of a $\mathrm{Fe} I$ line \\
\hline 687.8310 & 1.798 & -0.240 & only in V1261 Ori \\
\hline 689.2581 & 0.000 & -2.840 & only in V1261 Ori \\
\hline 707.0070 & 1.847 & -0.030 & \\
\hline Sr II & \multicolumn{2}{|c|}{$A_{\odot}(\mathrm{Sr})=2.92$} & \\
\hline 407.7719 & 0.000 & 0.170 & only in 14 Aur C \\
\hline 416.1792 & 2.940 & -0.600 & only in 14 Aur C and 63 Eri \\
\hline 421.5519 & 0.000 & -0.170 & only in 14 Aur C \\
\hline Y I & \multicolumn{2}{|c|}{$A_{\odot}(\mathrm{Y})=2.21$} & \\
\hline 563.0130 & 1.356 & 0.211 & only in IT Vir \\
\hline 613.8435 & 0.066 & -1.923 & \\
\hline 622.2578 & 0.000 & -1.452 & \\
\hline 640.2006 & 0.066 & -1.849 & only in 56 Peg and IT Vir \\
\hline 643.5004 & 0.066 & -0.820 & \\
\hline 668.7567 & 0.000 & -2.000 & only in V1261 Ori \\
\hline Y II & \multicolumn{2}{|c|}{$A_{\odot}(\mathrm{Y})=2.21$} & \\
\hline 395.0352 & 0.104 & -0.490 & only in 14 Aur C \\
\hline 488.3684 & 1.084 & 0.265 & only in 14 Aur C and V1379 Aql \\
\hline 490.0120 & 1.033 & 0.103 & only in IP Eri \\
\hline 508.7416 & 1.084 & -0.170 & \\
\hline 520.0406 & 0.992 & -0.570 & \\
\hline 528.9815 & 1.033 & -1.850 & \\
\hline 532.0782 & 1.084 & -1.950 & only in V1379 Aql \\
\hline 540.2774 & 1.839 & -0.630 & \\
\hline 554.4611 & 1.738 & -1.090 & only in 63 Eri and V1379 Aql \\
\hline 554.6009 & 1.748 & -0.754 & \\
\hline 566.2925 & 1.944 & 0.384 & \\
\hline 572.8890 & 1.839 & -1.120 & \\
\hline 661.3733 & 1.748 & -0.848 & \\
\hline 679.5414 & 1.738 & -1.030 & \\
\hline 788.1881 & 1.839 & -0.570 & \\
\hline $\mathrm{Zr} \mathbf{I}$ & \multicolumn{2}{|c|}{$A_{\odot}(\mathrm{Zr})=2.58$} & \\
\hline 473.9480 & 0.651 & 0.230 & only in 63 Eri \\
\hline 477.2323 & 0.623 & 0.040 & \\
\hline 488.7750 & 0.730 & 1.000 & \\
\hline 538.5151 & 0.519 & -0.710 & \\
\hline 568.0 .92 & 0.543 & -1.700 & only in IT Vir \\
\hline 573.5690 & 0.000 & -2.240 & only in $\zeta$ Cap \\
\hline
\end{tabular}

Notes. A vertical mark spanning different spectral lines identifies hyperfine or isotopic components.
Table A.1. continued.

\begin{tabular}{|c|c|c|c|}
\hline$\lambda[\mathrm{nm}]$ & $\chi[\mathrm{eV}]$ & $\log g f$ & \\
\hline 612.7475 & 0.154 & -1.060 & \\
\hline 613.4585 & 0.000 & -1.280 & \\
\hline 614.0535 & 0.519 & -1.410 & \\
\hline 614.3252 & 0.071 & -1.100 & \\
\hline 644.5747 & 0.999 & -0.830 & \\
\hline 650.6437 & 0.633 & -2.110 & only in $\zeta$ Cap \\
\hline 699.0869 & 0.623 & -1.220 & \\
\hline 709.7774 & 0.687 & -0.570 & only in V1379 Aql \\
\hline 716.9130 & 0.730 & -0.880 & \\
\hline 733.6066 & 0.519 & -2.170 & only in IT Vir \\
\hline 743.9889 & 0.543 & -1.810 & \\
\hline 755.3039 & 0.519 & -3.040 & only in V1261 Ori \\
\hline 755.4780 & 0.520 & -2.280 & only in V1261 Ori \\
\hline 755.8487 & 1.550 & -1.470 & only in V1261 Ori \\
\hline 760.7167 & 0.633 & -1.880 & \\
\hline 784.9365 & 0.687 & -1.300 & \\
\hline 805.8107 & 0.623 & -2.020 & only in IT Vir \\
\hline 806.3105 & 0.623 & -1.620 & only in $\zeta$ Cap \\
\hline 807.0115 & 0.730 & -0.790 & only in IP Eri and IT Vir \\
\hline 813.3011 & 0.687 & -1.130 & only in $\zeta$ Cap and IT Vir \\
\hline 846.4687 & 0.651 & -2.080 & only in IT Vir \\
\hline $\mathrm{Zr}$ II & $A_{\odot}(\mathrm{Zr})$ & $=2.58$ & \\
\hline 4150.986 & 0.802 & -0.992 & only in 14 Aur C \\
\hline 4211.877 & 0.527 & -1.040 & only in 14 Aur C \\
\hline 4379.742 & 1.532 & -0.356 & only in 14 Aur C \\
\hline 5112.270 & 1.665 & -0.850 & \\
\hline 5350.350 & 1.773 & -1.160 & \\
\hline 5418.016 & 1.756 & -1.600 & only in HR5692 \\
\hline Ba I & $A_{\odot}(\mathrm{Ba})$ & $=2.17$ & \\
\hline 748.8077 & 1.190 & -0.230 & \\
\hline Ba II & $A_{\odot}(\mathrm{Ba})$ & $=2.17$ & \\
\hline | $389.1776^{134}$ & 2.512 & 0.295 & \\
\hline $389.1776^{135}$ & 2.512 & 0.295 & \\
\hline $389.1776^{136}$ & 2.512 & 0.295 & Only in HR5692 \\
\hline $389.1776^{137}$ & 2.512 & 0.295 & \\
\hline $389.1776^{138}$ & 2.512 & 0.295 & \\
\hline $416.6000^{134}$ & 2.722 & -0.433 & \\
\hline $416.6000^{135}$ & 2.722 & -0.433 & \\
\hline $416.6000^{136}$ & 2.722 & -0.433 & Only in DR Dra \\
\hline $416.6000^{137}$ & 2.722 & -0.433 & \\
\hline $416.6000^{138}$ & 2.722 & -0.433 & \\
\hline | $452.4925^{130}$ & 2.512 & -0.390 & \\
\hline $452.4925^{132}$ & 2.512 & -0.390 & \\
\hline $452.4925^{134}$ & 2.512 & -0.390 & \\
\hline $452.4925^{135}$ & 2.512 & -0.390 & Only in 63 Eri \\
\hline $452.4925^{136}$ & 2.512 & -0.390 & \\
\hline $452.4925^{137}$ & 2.512 & -0.390 & \\
\hline $452.4925^{138}$ & 2.512 & -0.390 & \\
\hline |455.3998 & 0.000 & -0.666 & \\
\hline $455.3999^{137}$ & 0.000 & -0.666 & \\
\hline $455.4000^{137}$ & 0.000 & -1.064 & \\
\hline $455.4001^{135}$ & 0.000 & -0.666 & \\
\hline $455.4002^{135}$ & 0.000 & -1.064 & \\
\hline $455.4002^{135}$ & 0.000 & -0.666 & \\
\hline $455.4031^{130}$ & 0.000 & 0.140 & \\
\hline $455.4031^{132}$ & 0.000 & 0.140 & \\
\hline $455.4031^{134}$ & 0.000 & 0.140 & Only in 14 Aur C and V1379 Aql \\
\hline $455.4032^{136}$ & 0.000 & 0.140 & \\
\hline $455.4033^{138}$ & 0.000 & 0.140 & \\
\hline
\end{tabular}


T. Merle et al.: To Ba or not to Ba?

Table A.1. continued.

\begin{tabular}{|c|c|c|c|}
\hline$\lambda[\mathrm{nm}]$ & $\chi[\mathrm{eV}]$ & $\log g f$ & \\
\hline $455.4048^{135}$ & 0.000 & -0.219 & \\
\hline $455.4050^{135}$ & 0.000 & -0.666 & \\
\hline $455.4051^{137}$ & 0.000 & -0.219 & \\
\hline $455.4052^{135}$ & 0.000 & -1.365 & \\
\hline $455.4054^{137}$ & 0.000 & -0.666 & \\
\hline $455.4055^{137}$ & 0.000 & -1.365 & \\
\hline $493.4030^{137}$ & 0.000 & -0.662 & \\
\hline $493.4032^{135}$ & 0.000 & -0.662 & \\
\hline $493.4042^{135}$ & 0.000 & -1.361 & \\
\hline $493.4042^{137}$ & 0.000 & -1.361 & \\
\hline $493.4074^{130}$ & 0.000 & -0.157 & \\
\hline $493.4074^{132}$ & 0.000 & -0.157 & \\
\hline $493.4074^{134}$ & 0.000 & -0.157 & Only in 14 Aur C \\
\hline $493.4075^{136}$ & 0.000 & -0.157 & \\
\hline $493.4077^{138}$ & 0.000 & -0.157 & \\
\hline $493.4091^{135}$ & 0.000 & -0.662 & \\
\hline $493.4095^{137}$ & 0.000 & -0.662 & \\
\hline $493.4102^{135}$ & 0.000 & -0.662 & \\
\hline $493.4107^{137}$ & 0.000 & -0.662 & \\
\hline | $585.3669^{135}$ & 0.604 & -1.967 & \\
\hline $585.3669^{137}$ & 0.604 & -1.967 & \\
\hline $585.3670^{135}$ & 0.604 & -2.113 & \\
\hline $585.3670^{135}$ & 0.604 & -1.909 & \\
\hline $585.3671^{137}$ & 0.604 & -2.113 & \\
\hline $585.3671^{137}$ & 0.604 & -1.909 & \\
\hline $585.3672^{135}$ & 0.604 & -2.113 & \\
\hline $585.3672^{135}$ & 0.604 & -2.511 & \\
\hline $585.3673^{130}$ & 0.604 & -0.909 & \\
\hline $585.3673^{132}$ & 0.604 & -0.909 & \\
\hline $585.3673^{134}$ & 0.604 & -0.909 & \\
\hline $585.3673^{137}$ & 0.604 & -2.113 & \\
\hline $585.3673^{135}$ & 0.604 & -1.812 & \\
\hline $585.3673^{137}$ & 0.604 & -2.511 & \\
\hline $585.3674^{136}$ & 0.604 & -0.909 & \\
\hline $585.3675^{135}$ & 0.604 & -1.909 & \\
\hline $585.3675^{135}$ & 0.604 & -1.365 & \\
\hline $585.3675^{137}$ & 0.604 & -1.812 & \\
\hline $585.3675^{138}$ & 0.604 & -0.909 & \\
\hline $585.3676^{137}$ & 0.604 & -1.909 & \\
\hline $585.3676^{137}$ & 0.604 & -1.365 & \\
\hline $585.3680^{135}$ & 0.604 & -1.967 & \\
\hline $585.3682^{137}$ & 0.604 & -1.967 & \\
\hline $614.1708^{135}$ & 0.704 & -0.456 & \\
\hline $614.1708^{135}$ & 0.704 & -1.264 & \\
\hline $614.1709^{135}$ & 0.704 & -2.410 & \\
\hline $614.1709^{137}$ & 0.704 & -1.264 & \\
\hline $614.1709^{137}$ & 0.704 & -0.456 & \\
\hline $614.1710^{137}$ & 0.704 & -2.410 & \\
\hline $614.1711^{130}$ & 0.704 & -0.030 & \\
\hline $614.1711^{132}$ & 0.704 & -0.030 & \\
\hline $614.1711^{134}$ & 0.704 & -0.030 & \\
\hline $614.1712^{136}$ & 0.704 & -0.030 & \\
\hline $614.1713^{135}$ & 0.704 & -0.662 & \\
\hline $614.1713^{138}$ & 0.704 & -0.030 & \\
\hline $614.1714^{135}$ & 0.704 & -1.167 & \\
\hline $614.1715^{135}$ & 0.704 & -2.234 & \\
\hline $614.1715^{137}$ & 0.704 & -0.662 & \\
\hline $614.1716^{135}$ & 0.704 & -0.912 & \\
\hline $614.1716^{137}$ & 0.704 & -1.167 & \\
\hline $614.1717^{135}$ & 0.704 & -1.234 & \\
\hline $614.1717^{135}$ & 0.704 & -1.280 & \\
\hline $614.1717^{137}$ & 0.704 & -2.234 & \\
\hline
\end{tabular}

Table A.1. continued.

\begin{tabular}{|c|c|c|c|}
\hline$\lambda[\mathrm{nm}]$ & $\chi[\mathrm{eV}]$ & $\log g f$ & \\
\hline $614.1718^{137}$ & 0.704 & -0.912 & \\
\hline $614.1719^{137}$ & 0.704 & -1.234 & \\
\hline $614.1719^{137}$ & 0.704 & -1.280 & \\
\hline $649.6883^{135}$ & 0.604 & -1.911 & \\
\hline $649.6883^{137}$ & 0.604 & -1.911 & \\
\hline $649.6888^{135}$ & 0.604 & -1.212 & \\
\hline $649.6888^{137}$ & 0.604 & -1.212 & \\
\hline $649.6895^{130}$ & 0.604 & -0.406 & \\
\hline $649.6895^{132}$ & 0.604 & -0.406 & \\
\hline $649.6895^{134}$ & 0.604 & -0.406 & \\
\hline $649.6895^{135}$ & 0.604 & -0.765 & \\
\hline $649.6896^{137}$ & 0.604 & -0.765 & \\
\hline $649.6897^{136}$ & 0.604 & -0.406 & \\
\hline $649.6898^{138}$ & 0.604 & -0.406 & \\
\hline $649.6900^{135}$ & 0.604 & -1.610 & \\
\hline $649.6902^{135}$ & 0.604 & -1.212 & \\
\hline $649.6902^{137}$ & 0.604 & -1.610 & \\
\hline $649.6904^{137}$ & 0.604 & -1.212 & \\
\hline $649.6906^{135}$ & 0.604 & -1.212 & \\
\hline $649.6909^{137}$ & 0.604 & -1.212 & \\
\hline La II & $A_{\odot}(\mathrm{La})$ & 1.13 & \\
\hline | 394.9037 & 0.403 & -1.337 & \\
\hline 394.9038 & 0.403 & -1.191 & \\
\hline 394.9044 & 0.403 & -0.995 & \\
\hline 394.9046 & 0.403 & -1.008 & \\
\hline 394.9047 & 0.403 & -1.668 & \\
\hline 394.9056 & 0.403 & -0.761 & \\
\hline 394.9058 & 0.403 & -0.886 & \\
\hline 394.9059 & 0.403 & -1.559 & \\
\hline 394.9072 & 0.403 & -0.576 & \\
\hline 394.9075 & 0.403 & -0.825 & Only in 14 Aur C \\
\hline 394.9077 & 0.403 & -1.580 & \\
\hline 394.9093 & 0.403 & -0.420 & \\
\hline 394.9097 & 0.403 & -0.821 & \\
\hline 394.9100 & 0.403 & -1.690 & \\
\hline 394.9120 & 0.403 & -0.284 & \\
\hline 394.9124 & 0.403 & -0.887 & \\
\hline 394.9127 & 0.403 & -1.902 & \\
\hline 394.9151 & 0.403 & -0.163 & \\
\hline 394.9156 & 0.403 & -1.092 & \\
\hline 394.9160 & 0.403 & -2.305 & \\
\hline |398.8445 & 0.403 & -1.362 & \\
\hline 398.8446 & 0.403 & -1.839 & \\
\hline 398.8452 & 0.403 & -1.140 & \\
\hline 398.8454 & 0.403 & -1.362 & \\
\hline 398.8464 & 0.403 & -1.041 & \\
\hline 398.8466 & 0.403 & -1.985 & \\
\hline 398.8467 & 0.403 & -1.140 & \\
\hline 398.8482 & 0.403 & -1.015 & \\
\hline 398.8484 & 0.403 & -1.355 & \\
\hline 398.8486 & 0.403 & -1.041 & Only in 63 Eri \\
\hline 398.8504 & 0.403 & -1.068 & \\
\hline 398.8507 & 0.403 & -0.969 & \\
\hline 398.8509 & 0.403 & -1.015 & \\
\hline 398.8532 & 0.403 & -1.263 & \\
\hline 398.8535 & 0.403 & -0.683 & \\
\hline 398.8538 & 0.403 & -1.068 & \\
\hline 398.8569 & 0.403 & -0.455 & \\
\hline 398.8572 & 0.403 & -1.263 & \\
\hline 404.2901 & 0.927 & 0.290 & Only in 63 Eri \\
\hline 408.6695 & 0.000 & -1.266 & \\
\hline
\end{tabular}


Table A.1. continued.

\begin{tabular}{|c|c|c|c|}
\hline$\lambda[\mathrm{nm}]$ & $\chi[\mathrm{eV}]$ & $\log g f$ & \\
\hline 408.6699 & 0.000 & -1.108 & \\
\hline 408.6702 & 0.000 & -1.119 & \\
\hline 408.6705 & 0.000 & -1.292 & \\
\hline 408.6708 & 0.000 & -0.696 & \\
\hline 408.6709 & 0.000 & -1.094 & \\
\hline 408.6710 & 0.000 & -1.790 & Only in HR 5692 \\
\hline 408.6711 & 0.000 & -1.468 & \\
\hline 408.6711 & 0.000 & -3.216 & \\
\hline 408.6717 & 0.000 & -1.292 & \\
\hline 408.6719 & 0.000 & -1.119 & \\
\hline 408.6720 & 0.000 & -1.108 & \\
\hline 408.6721 & 0.000 & -1.266 & \\
\hline |466.2478 & 0.000 & -2.952 & \\
\hline 466.2482 & 0.000 & -2.511 & \\
\hline 466.2486 & 0.000 & -2.240 & \\
\hline 466.2491 & 0.000 & -2.253 & \\
\hline 466.2492 & 0.000 & -2.137 & \\
\hline 466.2493 & 0.000 & -2.256 & \\
\hline 466.2503 & 0.000 & -2.511 & \\
\hline 466.2505 & 0.000 & -2.056 & \\
\hline 466.2507 & 0.000 & -1.763 & \\
\hline 474.8726 & 0.927 & -0.540 & Only in $\zeta$ Cyg and 63 Eri \\
\hline 480.4039 & 0.235 & -1.490 & Only in $56 \mathrm{Peg}$ and IT vir \\
\hline |492.1774 & 0.244 & -1.139 & \\
\hline 492.1774 & 0.244 & -2.220 & \\
\hline 492.1774 & 0.244 & -3.601 & \\
\hline 492.1775 & 0.244 & -1.233 & \\
\hline 492.1775 & 0.244 & -2.005 & \\
\hline 492.1775 & 0.244 & -3.207 & \\
\hline 492.1776 & 0.244 & -1.334 & \\
\hline 492.1776 & 0.244 & -1.445 & \\
\hline 492.1776 & 0.244 & -1.915 & \\
\hline 492.1776 & 0.244 & -1.927 & \\
\hline 492.1776 & 0.244 & -2.923 & Only in $\zeta$ Cap et 14 Aur C \\
\hline 492.1776 & 0.244 & -3.010 & \\
\hline 492.1777 & 0.244 & -1.566 & \\
\hline 492.1777 & 0.244 & -1.955 & \\
\hline 492.1777 & 0.244 & -2.939 & \\
\hline 492.1778 & 0.244 & -1.700 & \\
\hline 492.1778 & 0.244 & -1.848 & \\
\hline 492.1778 & 0.244 & -2.006 & \\
\hline 492.1778 & 0.244 & -2.053 & \\
\hline 492.1778 & 0.244 & -2.258 & \\
\hline 492.1778 & 0.244 & -3.123 & \\
\hline 511.4559 & 0.235 & -1.030 & Only in 14 Aur C \\
\hline 529.0818 & 0.000 & -1.650 & \\
\hline |530.1908 & 0.403 & -3.065 & \\
\hline 530.1913 & 0.403 & -2.266 & \\
\hline 530.1917 & 0.403 & -2.391 & \\
\hline 530.1946 & 0.403 & -3.483 & \\
\hline 530.1953 & 0.403 & -2.300 & \\
\hline 530.1958 & 0.403 & -2.120 & \\
\hline 530.2001 & 0.403 & -2.483 & \\
\hline 530.2008 & 0.403 & -1.913 & \\
\hline |530.2067 & 0.403 & -1.742 & \\
\hline 530.3513 & 0.321 & -1.874 & \\
\hline 530.3513 & 0.321 & -2.363 & \\
\hline 530.3514 & 0.321 & -3.062 & \\
\hline 530.3531 & 0.321 & -2.167 & \\
\hline 530.3532 & 0.321 & -2.247 & \\
\hline
\end{tabular}

Table A.1. continued.

\begin{tabular}{|c|c|c|c|}
\hline$\lambda[\mathrm{nm}]$ & $\chi[\mathrm{eV}]$ & $\log g f$ & \\
\hline 530.3532 & 0.321 & -2.622 & \\
\hline 530.3546 & 0.321 & -2.366 & \\
\hline 530.3546 & 0.321 & -2.622 & \\
\hline 530.3547 & 0.321 & -2.351 & \\
\hline 580.5773 & 0.126 & -1.560 & \\
\hline 586.3691 & 0.927 & -1.370 & Only in $\zeta$ Cap \\
\hline 593.6210 & 0.173 & -2.070 & \\
\hline 617.2721 & 0.126 & -2.253 & \\
\hline |626.2422 & 0.403 & -1.873 & \\
\hline 626.2429 & 0.403 & -2.802 & \\
\hline 626.2434 & 0.403 & -4.015 & \\
\hline 639.0455 & 0.321 & -2.012 & \\
\hline 639.0468 & 0.321 & -2.183 & \\
\hline 639.0468 & 0.321 & -2.752 & \\
\hline 639.0479 & 0.321 & -2.570 & \\
\hline 639.0479 & 0.321 & -3.752 & \\
\hline 639.0480 & 0.321 & -2.390 & \\
\hline 639.0489 & 0.321 & -2.536 & \\
\hline 639.0489 & 0.321 & -3.334 & \\
\hline 639.0490 & 0.321 & -2.661 & \\
\hline 639.0496 & 0.321 & -3.100 & \\
\hline 639.0497 & 0.321 & -2.595 & \\
\hline 639.0498 & 0.321 & -3.079 & \\
\hline 639.0502 & 0.321 & -2.954 & \\
\hline 639.0503 & 0.321 & -2.778 & \\
\hline 639.0506 & 0.321 & -2.857 & \\
\hline 677.4268 & 0.126 & -1.708 & \\
\hline 683.4099 & 0.244 & -2.168 & Only in $\zeta$ Cap and $56 \mathrm{Peg}$ \\
\hline 706.6198 & 0.000 & -1.655 & Only in IP Eri and DR Dra \\
\hline 748.3490 & 0.126 & -1.891 & \\
\hline Ce II & \multicolumn{2}{|c|}{$A_{\odot}(\mathrm{Ce})=1.70$} & \\
\hline 434.9768 & 0.529 & -0.520 & \\
\hline 434.9789 & 0.701 & -0.350 & \\
\hline 451.5848 & 1.058 & -0.240 & Only in $\zeta$ Cyg and HR 5692 \\
\hline 456.2359 & 0.478 & 0.230 & \\
\hline 462.8239 & 1.366 & -0.430 & \\
\hline 477.3941 & 0.924 & -0.390 & Only in $\zeta$ Cap \\
\hline 494.3441 & 1.206 & -0.360 & \\
\hline 494.4618 & 1.008 & -0.520 & Only in $\zeta$ Cap \\
\hline 518.7503 & 0.559 & -3.330 & \\
\hline 527.4229 & 1.044 & 0.130 & Only in $\zeta$ Cap and 14 Aur \\
\hline 533.0556 & 0.869 & -0.400 & \\
\hline 5472.279 & 1.247 & -0.100 & Only in 63 Eri \\
\hline 597.5818 & 1.327 & -0.460 & Only in IP Eri \\
\hline 597.5881 & 1.251 & -1.680 & and HR 5692 \\
\hline 604.3373 & 1.206 & -0.480 & \\
\hline 605.1815 & 0.232 & -1.530 & \\
\hline 605.2617 & 1.838 & -0.800 & Only in $\zeta$ Cap \\
\hline 758.0913 & 0.327 & -2.120 & Only in V1261 Ori \\
\hline 802.5571 & 0.000 & -1.420 & \\
\hline 871.6659 & 0.122 & -1.980 & \\
\hline 877.2135 & 0.357 & -1.260 & \\
\hline 891.0948 & 0.435 & -1.800 & \\
\hline 897.0176 & 0.295 & -2.020 & Only in V1261 Ori \\
\hline 899.3403 & 0.265 & -2.520 & Only in V1261 Ori \\
\hline
\end{tabular}


T. Merle et al.: To Ba or not to $\mathrm{Ba}$ ?

\section{Appendix B: Molecular line list}

Table B.1. Molecular line list.

\begin{tabular}{ll}
\hline \hline$\lambda[\mathrm{nm}]$ & Comments \\
\hline $\mathbf{C}_{2}$ & \\
468.427 & only used for 14 Aur C \\
471.30 & \\
473.277 & \\
473.50 & \\
473.531 & \\
489.748 & region difficult to fit \\
493.667 & \\
495.142 & \\
495.60 & \\
496.363 & \\
502.416 & \\
503.36 & \\
505.88 & \\
506.672 & \\
507.35 & \\
507.528 & \\
508.92 & \\
513.56 & used in Barbuy et al. (1992) \\
514.12 & \\
558.52 & \\
562.12 &
\end{tabular}

\begin{tabular}{l} 
CN \\
639.63 \\
642.07 \\
645.85 \\
647.72 \\
651.97 \\
653.56 \\
654.90 \\
696.19 \\
697.42 \\
700.91 \\
700.96 \\
701.19 \\
720.52 \\
723.81 \\
726.36 \\
733.70 \\
734.01 \\
734.06 \\
734.23 \\
737.45 \\
738.45 \\
739.79 \\
740.34 \\
745.63 \\
749.92 \\
753.95 \\
754.91 \\
757.19 \\
787.40 \\
789.21 \\
790.67 \\
790.96 \\
791.18 \\
792.30 \\
792.97 \\
793.86 \\
793.92 \\
795.17 \\
795.41 \\
795.82 \\
796.71 \\
\hline
\end{tabular}

Table B.1. continued.

\begin{tabular}{ll}
\hline \hline$\lambda[\mathrm{nm}]$ & Comments \\
\hline 797.08 \\
797.38 \\
797.47 \\
797.72 \\
797.98 \\
798.11 \\
798.46 \\
798.52 \\
799.23 \\
799.56 \\
800.85 \\
801.57 \\
801.70 \\
801.81 \\
803.28 \\
803.50 \\
803.81 \\
804.17 \\
805.31 \\
805.73 \\
806.41 \\
807.44 \\
808.25 \\
808.62 \\
808.73 \\
808.83 \\
810.04 \\
810.15 \\
811.87 \\
812.40 \\
812.56 \\
813.21 \\
816.01 \\
816.69 \\
817.51 \\
818.02 \\
820.70 \\
823.60 \\
824.73 \\
826.72 \\
826.80 \\
829.12 \\
831.24 \\
832.55 \\
833.04 \\
833.74 \\
835.07 \\
837.15 \\
839.12 \\
839.85 \\
840.72 \\
841.13 \\
842.05 \\
842.24 \\
843.00 \\
843.12 \\
844.18 \\
846.99 \\
847.09 \\
847.24 \\
847.84 \\
850.51 \\
852.34 \\
856.70 \\
857.33 \\
\hline
\end{tabular}


A\&A 586, A151 (2016)

Table B.2. continued.

\begin{tabular}{ll}
\hline \hline$\lambda[\mathrm{nm}]$ & Comments \\
\hline 857.72 & \\
858.70 & \\
860.49 & \\
860.77 & \\
862.05 & \\
862.44 & \\
863.00 & \\
862.91 & \\
870.31 & \\
870.68 & \\
875.97 & \\
881.62 & \\
881.79 & \\
884.19 & \\
\hline
\end{tabular}

